\title{
A Model to Study NMDA Receptors in Early Nervous System Development
}

\author{
Josiah D. Zoodsma, ${ }^{1,3^{*}}$ Kelvin Chan, ${ }^{1,2,3^{*}}$ Ashwin A. Bhandiwad, ${ }^{6}$ David R. Golann, ${ }^{3}$ Guangmei Liu, ${ }^{4}$ \\ Shoaib A. Syed, ${ }^{3}$ Amalia J. Napoli, ${ }^{1,3}$ Harold A. Burgess, ${ }^{6}{ }^{\circledR}$ Howard I. Sirotkin, ${ }^{3 \dagger}$ and ${ }^{\circledR}$ Lonnie P. Wollmuth ${ }^{3,4,5 \dagger}$ \\ ${ }^{1}$ Graduate Program in Neuroscience, ${ }^{2}$ Medical Scientist Training Program, ${ }^{3}$ Department of Neurobiology \& Behavior, ${ }^{4}$ Department of Biochemistry \\ \& Cell Biology, ${ }^{5}$ Center for Nervous System Disorders, Stony Brook University, Stony Brook, New York 11794-5230, and ${ }^{6}$ Division of \\ Developmental Biology, Eunice Kennedy Shriver National Institute of Child Health and Human Development, Bethesda, Maryland
}

$\mathrm{N}$-methyl-D-aspartate receptors (NMDARs) are glutamate-gated ion channels that play critical roles in neuronal development and nervous system function. Here, we developed a model to study NMDARs in early development in zebrafish, by generating CRISPR-mediated lesions in the NMDAR genes, grinla and grin1b, which encode the obligatory GluN1 subunits. While receptors containing grin1a or grin $1 b$ show high $\mathrm{Ca}^{2+}$ permeability, like their mammalian counterpart, grin1a is expressed earlier

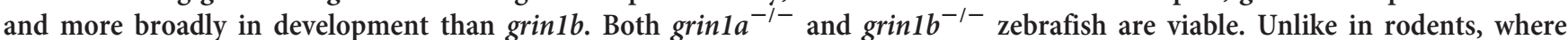
the grin1 knockout is embryonic lethal, grin1 double-mutant fish $\left(\right.$ grin1 $a^{-/-}$; grin1 $\left.1 b^{-/-}\right)$, which lack all NMDAR-mediated synaptic transmission, survive until $\sim 10 \mathrm{~d} \mathrm{dpf}$ (days post fertilization), providing a unique opportunity to explore NMDAR function during development and in generating behaviors. Many behavioral defects in the grin1 double-mutant larvae, including abnormal evoked responses to light and acoustic stimuli, prey-capture deficits, and a failure to habituate to acoustic stimuli, are replicated by short-term treatment with the NMDAR antagonist MK-801, suggesting that they arise from acute effects of compromised NMDAR-mediated transmission. Other defects, however, such as periods of hyperactivity and alterations in place preference, are not phenocopied by MK-801, suggesting a developmental origin. Together, we have developed a unique model to study NMDARs in the developing vertebrate nervous system.

Key words: calcium permeability; CRISPR-Cas9; habituation; prey-capture; visual acuity; zebrafish

Significance Statement

Rapid communication between cells in the nervous system depends on ion channels that are directly activated by chemical neurotransmitters. One such ligand-gated ion channel, the NMDAR, impacts nearly all forms of nervous system function. It has been challenging, however, to study the prolonged absence of NMDARs in vertebrates, and hence their role in nervous system development, due to experimental limitations. Here, we demonstrate that zebrafish lacking all NMDAR transmission are viable through early development and are capable of a wide range of stereotypic behaviors. As such, this zebrafish model provides a unique opportunity to study the role of NMDAR in the development of the early vertebrate nervous system.

Received Dec. 22, 2019; revised Mar. 5, 2020; accepted Mar. 12, 2020.

Author contributions: J.D.Z., K.C., A.A.B., D.R.G., H.A.B., H.I.S., and L.P.W. designed research; J.D.Z., K.C., A.A.B., D.R.G., G.L., S.A.S., and A.J.N. performed research; J.D.Z., K.C., A.A.B., D.R.G., G.L., S.A.S., A.J.N., H.A.B., H.I.S., and L.P.W. analyzed data; J.D.Z., K.C., H.I.S., and L.P.W. wrote the first draft of the paper; J.D.Z., K.C., A.A.B., H.A.B., H.I.S., and L.P.W. edited the paper; H.I.S. and L.P.W. wrote the paper.

This work was supported by National Institutes of Health Grants National Research Service Award F30MH115618 to K.C. and R01EY003821 to L.P.W., Stony Brook University Targeted Research Opportunity Grant to L.P.W. and H.I.S., and Hudson River Foundation Grant to H.I.S. We thank Dr. Jane Cox for the grin1 probes; Donna Schmidt, Diane Henry-Vanisko, Abby Frenkel, and Julianna Casella for technical assistance; Stephanie Flanagan, Neal Bhattacharji, and the undergraduate fish room aides for fish care; and the Intramural Research Program of the Eunice Kennedy Shriver National Institute for Child Health and Human Development.

The authors declare no competing financial interests.

*J.D.Z. and K.C. contributed equally to this work.

†H.I.S. and L.P.W. contributed equally to this work as cosenior authors.

Correspondence should be addressed to Lonnie P. Wollmuth at lonnie.wollmuth@stonybrook.edu.

https://doi.org/10.1523/JNEUROSCI.3025-19.2020

Copyright $\odot 2020$ the authors

\section{Introduction}

Nervous system function depends on the development of brain circuits that integrate appropriate cell types and establish proper connectivity. In the vertebrate nervous system, glutamate is the major excitatory neurotransmitter. At synapses that mediate rapid transmission, glutamate is converted into biological signals by ligand-gated or ionotropic glutamate receptors (iGluRs), including AMPAR, kainate, and NMDAR subtypes (Traynelis et al., 2010). Because of its unique signaling properties, including high $\mathrm{Ca}^{2+}$ permeability, NMDARs are central to higher brain functions, such as the plasticity underlining learning and memory (Hunt and Castillo, 2012; Paoletti et al., 2013) and brain development (Cline and Haas, 2008; Gambrill and Barria, 2011; Chakraborty et al., 2017). Because of the experimental limitations of pharmacological manipulation, especially in studies viewing 
long-term outcomes as occurs for many neurodevelopmental disorders, studying NMDARs in early circuit development is challenging. Adding to this challenge, the loss of NMDAR subunits critical to early brain development in murine models is embryonic or perinatal lethal (Forrest et al., 1994; Kutsuwada et al., 1996; Sprengel et al., 1998).

Zebrafish are a powerful model to study early nervous system development as fertilization is external and larvae are transparent, which facilitates imaging of cell migration and circuit formation. Furthermore, zebrafish larvae exhibit complex behaviors that provide simple and robust readouts of higher nervous system function. Previous studies of NMDARs in zebrafish have generally used acute pharmacological manipulations, mainly MK-801, a specific antagonist of NMDARs (Huettner and Bean, 1988; Traynelis et al., 2010). These studies have highlighted the central role of NMDARs in complex brain functions and behaviors, including associative learning (Sison and Gerlai, 2011b), social interactions (Seibt et al., 2011; Dreosti et al., 2015), place preference (Swain et al., 2004), and responses to acoustic stimuli and prepulse inhibition (PPI) (Bergeron et al., 2015). Although these studies are invaluable, they do not reveal how brain or circuit development impacts specific behaviors because acute antagonist applications only change brain function for a short developmental time point and can have off-target effects.

NMDARs are heterotetramers composed of two obligate GluN1 subunits and typically two GluN2 (A-D) subunits (Paoletti et al., 2013). In zebrafish, the obligatory GluN1 subunit is encoded by two paralogous genes: grin1a and grin1b. The expression patterns of these genes have only been described before 2 days post fertilization (dpf) (Cox et al., 2005), and functional differences between paralogs are unknown. Here, we studied the developmental expression of grin $1 a$ and grin $1 b$ and the effect of their knockouts on early nervous system function. While their encoded proteins, GluN1a and GluN1b, show high $\mathrm{Ca}^{2+}$ permeability, like their mammalian counterparts, grin1a is expressed earlier in development and tends to be more widely expressed. Nevertheless, both grin $1 a^{-1-}$ and grin $1 b^{-1-}$ fish are viable as adults. Unlike rodents, grin1 double-mutant fish $\left(\right.$ grin $1 a^{-1-}$; grin $\left.1 b^{-1-}\right)$, which lack all NMDAR-mediated synaptic transmission, survive until $\sim 10 \mathrm{dpf}$, which is old enough to assess the impact of NMDAR deficits on larval behaviors. We took advantage of these fish to study the role of NMDARs in the development of numerous complex behaviors, including preycapture, spontaneous and evoked movements, habituation, and PPI.

\section{Materials and Methods}

Whole-cell electrophysiology

Human embryonic kidney 293 (HEK293) cells were grown in DMEM, supplemented with $10 \%$ FBS, for $24 \mathrm{~h}$ before transfection (Yelshansky et al., 2004; Alsaloum et al., 2016). Zebrafish cDNA constructs were synthesized from GenScript in a pcDNA3.1(+)-p2A-eGFP vector. Zebrafish (zGluN1a/b) and rat (rGluN2A) NMDAR-encoding cDNA constructs, were cotransfected into HEK293 cells along with a separate peGFP-Cl construct at a ratio of 4:4:1 (N1:N2:eGFP) for whole-cell or macroscopic recordings using X-tremeGene HP (Roche Diagnostics, 06-366). HEK293 cells were bathed in medium containing the GluN2 competitive antagonist DL-2-amino-5-phosphopentanoic acid (100 $\mu \mathrm{M}$, Tocris Bioscience) and $\mathrm{Mg}^{2+}(100 \mu \mathrm{M})$. All experiments were performed 24-48 $\mathrm{h}$ after transfection.

Whole-cell currents were recorded at room temperature $\left(20^{\circ} \mathrm{C}-23^{\circ} \mathrm{C}\right)$ using an EPC-10 amplifier with PatchMaster software (HEKA), digitized at $10 \mathrm{kHz}$, and low-pass filtered at $2.9 \mathrm{kHz}(-3 \mathrm{~dB})$ using an 8 pole low pass Bessel filter 4 (Yelshansky et al., 2004; Alsaloum et al., 2016). Patch microelectrodes were filled with an intracellular solution (in $\mathrm{mM}$ as follows): $140 \mathrm{KCl}, 10 \mathrm{HEPES}, 1 \mathrm{BAPTA}, 4 \mathrm{Mg}^{2+}$-ATP, $0.3 \mathrm{Na}^{+}$-GTP, pH 7.3 (KOH), $297 \mathrm{mOsm}$ (sucrose). Our standard extracellular solution consisted of the following (mM): $150 \mathrm{NaCl}, 2.5 \mathrm{KCl}$, and $10 \mathrm{HEPES}, \mathrm{pH}$ $7.2(\mathrm{NaOH})$. Currents were measured within $10 \mathrm{~min}$ of going whole cell.

External solutions were applied using a piezo-driven double-barrel application system. For agonist application, one barrel contained the external solution $0.1 \mathrm{~mm}$ glycine, whereas the other barrel contained both $0.1 \mathrm{~mm}$ glycine and $1 \mathrm{~mm}$ glutamate. For display, NMDAR currents were digitally refiltered at $500 \mathrm{~Hz}$ and resampled at $1 \mathrm{kHz}$.

\section{Zebrafish maintenance and housing}

Adult zebrafish strains were maintained at $28.5^{\circ} \mathrm{C}$ under a $13: 11 \mathrm{~h} \mathrm{light}$ to dark cycle and were fed a diet of artemia and GEMMA micropellets. The WT strain used for all experiments was a hybrid WT background consisting of Tubingen long-fin crossed to Brian's WT. The experiments and procedures were approved by the Stony Brook University Institutional Animal Care and Use Committee.

Whole-mount RNA in situ hybridization (ISH)

The grinla probe was previously described (Cox et al., 2005). Probes for grin1b were generated by PCR amplification of adult brain WT cDNA using the following primers: grinlb: forward (GAGtatttaggtgacactatag TCTGTGGACTAGCTGGCAAA) and reverse (GAGtaatacgactcacta tagggATGGACGTTGCGTGTTTGTA).

Lowercase letters in forward and reverse primers indicate the T7 and SP6 RNA polymerase binding sites, respectively. DIG-labeled antisense and sense probes were generated from PCR product.

Whole-mount RNA ISH was performed as described by Thisse et al. (1993). Embryos were stained with NBT/BCIP. Before photography, embryos were cleared with 2:1 benzyl benzoate to benzyl alcohol and then mounted in Canada Balsam with 2.5\% methyl salicyclate. Embryos were imaged on a Zeiss Axioplan 2 microscope with Axiocam HRc mounted camera and AxioVision Software.

\section{CRISPR-Cas 9 gene targeting and zebrafish microinjections}

gRNAs were designed through IDT custom gRNA Design Tool. gRNAs were complexed to Cas9 protein, forming a ribonucleoprotein, using the Alt-R CRISPR-Cas9 System (IDT); $0.5 \mathrm{nl}$ of ribonucleoprotein (25 pg of gRNA and 125 pg of Cas9 protein) was injected into the cell of pronased $(1 \mathrm{mg} / \mathrm{ml}) 1$-cell embryos. Ten to 20 embryos from each injection were assayed (by PCR) for lesions using primers flanking the gRNA target site. If insertions or deletions were detected when screened on a conventional agarose gel, siblings were grown to adulthood and screened for germline transmission. All mutations were outcrossed at least twice to minimize the effect of off-target endonuclease activity on behavioral phenotypes.

\section{Zebrafish genotyping and size comparisons}

Adult zebrafish viability was assayed after 2 months post-fertilization. The following primers were used to screen the CRISPR mutations by PCR and for subsequent genotyping: grin1a: forward (ATTAGGAATG GTGTGGGCTGGC) and reverse (GGTGATGCGCTCCTCAGGCC); and grin $1 b$ : forward (GGTGCCCCTCGGAGCTTTTC) and reverse (GGAAGGCTGCCAAATTGGCAGT).

For size comparisons of adult zebrafish, sibling WT and mutant fish were generated from a heterozygous intercross and raised together in the same tanks, with equal densities across the different tanks. Size measurements were reported as length from the snout tip to the fork of the caudal fin. Genotyping and establishment of sex were not performed until after measurements at 2 months. Results were reported as a combination of both male and female fish, as separation of fish by sex did not alter the reported results.

\section{$R T-P C R$}

RNA was extracted from pools of four larvae at $3 \mathrm{dpf}$ from homozygous mutant or homozygous WT intercrosses using Trizol (Invitrogen) and Direct-zol RNA Minipreps (Zymo Research). cDNA was synthesized from $0.2 \mu \mathrm{g}$ of RNA using Superscript II Reverse Transcriptase 
A rGluN1/rGluN2A

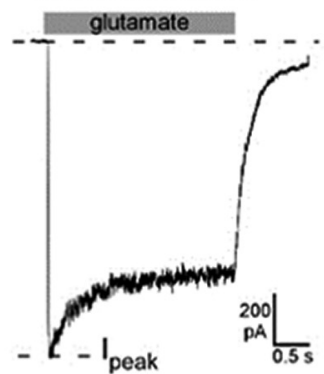

C

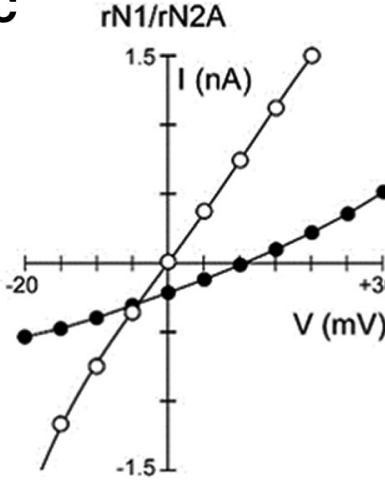

zGluN1a/rGluN2A

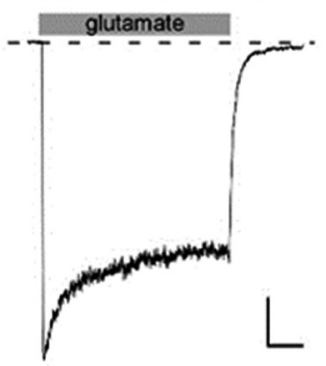

$\mathrm{zN} 1 \mathrm{a} / \mathrm{rN} 2 \mathrm{~A}$

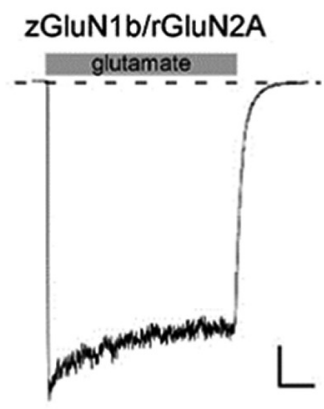

B
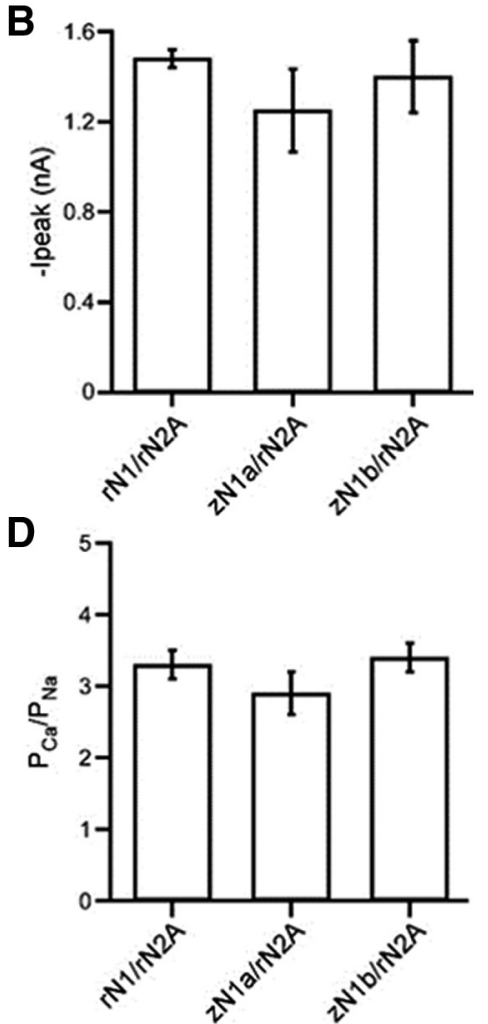

Figure 1. Zebrafish paralogs show functional currents and high $\mathrm{Ca}^{2+}$ permeability. $\boldsymbol{A}$, Whole-cell glutamate-activated currents recorded in HEK293 cells in response to sustained (2.5 s) glutamate applications (1 mM, gray bar) to measure NMDAR currents for various GluN1 constructs: rat GluN1 (rGluN1) (left); and zebrafish GluN1a (zGluN1a) (middle) or GluN1b (zGluN1b) (right). All GluN1 constructs were coexpressed with rat GluN2A (rGluN2A). Peak current amplitudes $\left(I_{\text {peak }}\right)$ were measured at the beginning of the glutamate application. Holding potential, $-70 \mathrm{mV}$.

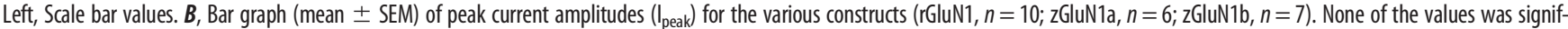
icantly different $\left(F_{(2,20)}=0.84, p=0.445\right.$, ANOVA). C, Measuring $\mathrm{Ca}^{2+}$ permeability. Current-voltage (I-V) relationships in an external solution containing high $\mathrm{Na}^{+}(140 \mathrm{~mm})$ and 0 added $\mathrm{Ca}^{2+}$ (open circles) or $10 \mathrm{~mm} \mathrm{Ca}{ }^{2+}$ (solid circles) to determine reversal potentials $\left(E_{\text {rev }}\right)$, the point where currents cross the 0 current axis. The $0 \mathrm{Ca}^{2+} \mathrm{I}-\mathrm{V}$ is the average of that recorded before and after the $10 \mathrm{~mm} \mathrm{Ca}{ }^{2+}$ recording. We used changes in reversal potentials $\left(\Delta \mathrm{E}_{\mathrm{rev}}\right)$ to calculate $\mathrm{P}_{\mathrm{Ca}_{2}} / \mathrm{P}_{\mathrm{Na}}$ (Jatzke et al., 2002). D, Relative calcium permeability $\left(\mathrm{P}_{\mathrm{Ca}} / \mathrm{P}_{\mathrm{Na}}\right)(\mathrm{mean} \pm \mathrm{SEM})$ calculated from $\Delta \mathrm{E}_{\text {rev }}$ for rat rGluN1/rGluN2A $(8.3 \pm 0.4 \mathrm{mV}, n=8)$, zGluN1a/rGluN2A $(7.9 \pm 0.7 \mathrm{mV}, n=9)$, or zGluN1b/rGluN2A $(8.6 \pm 0.5 \mathrm{mV}, n=8)$. None of the values was significantly different $\left(F_{(2,22)}=0.99, p=0.39\right.$, ANOVA).

(Invitrogen). The following primers were used to screen the cDNA by PCR: grin1a: forward (ATTAGGAATGGTGTGGGCTGGC) and reverse (ATGAATTTGTCTGATGGGTTCCT); and grin1b: forward (GTGCCCCTCGGAGCTTTTCG) and reverse (AGGAAGGCTGCCA AATTGGCA).

$q P C R$

RNA was extracted as stated above. Pools of four larvae were collected at 1 and $3 \mathrm{dpf}$, while pools of 2 larvae were collected at $5 \mathrm{dpf}$. qPCR was conducted on a LightCycler 480 (Roche Diagnostics) using PerfeCTa SYBR GreenFastMix (QuantaBio). Transcript levels from each sample were normalized to $\beta$-actin. In each experiment, three pools of cDNA from biological replicates were run in duplicate for each genotype. The following primers were used to screen the cDNA by PCR (Menezes et al., 2015): grin1a: forward (ATAAAGACGCCCGCAGGAAGC) and reverse (CGTGCTGACAGACGGGTCCGAC); and grin1b: forward (AATGCAGCTGGCCTTTGCAGC) and reverse(CTCTTGATGTTGG AGGCCAGGTTG).

Live imaging

Zebrafish larvae were immersed in 3\% methylcellulose, after anesthetization in ice water, and images were taken on a Zeiss Discovery version 20.

\section{Western blot}

Total protein was isolated from $7 \mathrm{dpf}$ zebrafish heads from WT and grin1 double mutants, and HEK293 cells transfected with plasmids encoding zGluN1a and zGluN1b, along with rat GluN2A. grin1 double- mutant numbers were enhanced through a behavior screen at $6 \mathrm{dpf}$, isolating $\sim 85 \%$ grin 1 double mutants. Post hoc genotyping confirmed this recovery rate. Twenty-four heads were isolated from each genotype and placed into $80 \mu \mathrm{l}$ of lysis buffer consisting of $1 \times$ Cell Lysis Buffer (Cell Signaling Technology, \#9803), $1 \mathrm{~mm}$ PMSF, and 1 tablet of cOmplete protease inhibitor cocktail tablet (Sigma Millipore, \#11836170001). For each HEK293 sample, a confluent $60 \mathrm{~mm}$ plate of HEK293 cells was isolated with $500 \mu \mathrm{l}$ of lysis buffer. Samples were sonicated (model: Lab Supplies, G112SPIT, $80 \mathrm{kHz}, 600 \mathrm{~V}$ ) for $2 \mathrm{~min}$ on ice-cold water and subsequently were incubated for $15 \mathrm{~min}$ at $4^{\circ} \mathrm{C}$, and centrifuged at $14,000 \mathrm{rpm}, 15 \mathrm{~min}$ at $4^{\circ} \mathrm{C}$ (model: Eppendorf Centrifuge 5417R; rotor: 45-30-11). HEK293 protein sample concentrations were quantified using a DC Lowry Assay kit (Bio-Rad, \#500-0116). Zebrafish protein samples could not be quantified since the absorbance was below the detection threshold; $20 \mu$ of zebrafish lysate or $50 \mu \mathrm{g}$ of HEK293 total protein was boiled in $2 \times$ Laemmli's buffer with $10 \% \beta$-mercaptoethanol for $10 \mathrm{~min}$ at $95^{\circ} \mathrm{C}$. Samples and Chameleon Duo IR Dye ladder (Li-Cor, \#92860000 ) were loaded onto Mini-PROTEAN TGX 7\% Precast Gel (Bio$\mathrm{Rad}$ ) and electrophoresed at $120 \mathrm{~V}$ for $60 \mathrm{~min}$. Gel was transferred to a methanol-activated PVDF membrane using the wet transfer system Mini Trans-Blot Cell (Bio-Rad).

PVDF membrane was washed with $\mathrm{dH}_{2} \mathrm{O}$, reactivated with methanol, then washed with $1 \times$ TBS. Membrane was incubated in Intercept blocking buffer (Li-Cor, \#927-70001) for $60 \mathrm{~min}$ at room temperature in dark. Membrane was incubated with primary antibodies in blocking buffer 
with $0.2 \%$ Tween-20: 1:500 mouse antiGluN1 (Synaptic Systems, \#114-001), 1:500 rabbit anti-GAPDH (Abcam, \#ab210113) for $60 \mathrm{~min}$ at room temperature in dark. Membrane was washed with $1 \times$ TBS with $0.1 \%$ Tween-20, then incubated with secondary antibodies in blocking buffer with $0.2 \%$ Tween-20, $0.02 \%$ SDS: 1:5000 donkey anti-mouse-IR800CW (Li-Cor, \#926-32212), 1:5000 goat anti-rabbit-IR800CW (Li-Cor, \#926-32211) for $60 \mathrm{~min}$ at room temperature in dark. Membrane was then washed with $1 \times$ TBS and imaged using Odyssey infrared imaging system(Li-Cor).

\section{Behavioral assays}

Behavioral assays were performed on multiple clutches from different parents to minimize the effects of genetic background. Embryos were kept in $150 \mathrm{~mm}$ Petri dishes in egg water $(6 \times g$ of synthetic sea salt, 20 $\mathrm{ml}$ of methylene blue $(1 \times g / \mathrm{L})$ solution in $20 \mathrm{~L}$ of water, $\mathrm{pH} 7$, at concentrations $<100$ larvae per plate.

Pharmacology. MK-801 (20 mM) was diluted in DMSO. For all experiments, unless otherwise noted, final concentration used was $20 \mu \mathrm{M}$ in $0.1 \%$ DMSO. The dosage of MK$801(20 \mu \mathrm{M})$ used throughout this study was an intermediate concentration from a previously performed dose-response analysis of MK-801 on zebrafish (Sison and Gerlai, 2011a).

Prey-capture paradigm. Paramecium multimicronucleatum were obtained from Carolina Biological Supply and were maintained at room temperature with new cultures generated every 3 weeks. Paramecia from cultures of 2-5 weeks of age were filtered and moved to a $35 \mathrm{~mm}$ Petri dish on the day of the assay.

Individual larvae were assayed at $7 \mathrm{dpf}$; 20-60 paramecia were added to individual 35 $\mathrm{mm}$ Petri dishes filled with $3 \mathrm{ml}$ of either system or egg water, yielding a water depth of $\sim 3 \mathrm{~mm}$. Fifty frames $(\sim 2.5 \mathrm{~s})$ of paramecia movement were recorded using an Excelis MPX5C-PRO camera with Mosaic version 2.0 software at the beginning and end of each trial, which lasted $1.5 \mathrm{~h}$. These videos were processed using ImageJ as previously described (Gahtan et al., 2005). Paramecia-trace images were manually counted by a blind viewer. Multiple control plates containing only paramecium were used in each assay to account for any decrease in paramecia viability. Results were expressed as a ratio of the proportion of paramecia eaten, normalized to their respective control.

Dark flash behavior paradigm. Dark-flash experiments were performed in 9-well gridded plates. Each plate of fish was light-adapted for 20 min before testing. Ten dark flash stimuli were administered with an interstimulus interval (ISI) of $1 \mathrm{~s}$ and a dark-flash consisting of a whitespectrum light $\left(\sim 500 \mu \mathrm{W} / \mathrm{cm}^{2}\right)$ turning off for $1 \mathrm{~s}$. Larvae were tracked for $600 \mathrm{~ms}$ at 1000 frames/s using a DRS Lightning high speed camera (DEL Imaging) to measure O-bend responsiveness. Kinematics were analyzed using FLOTE software (Burgess and Granato, 2007a).

Spontaneous and evoked movement paradigm. At $48 \mathrm{~h}$ post fertilization (hpf), embryos were transitioned to 24-well behavior plates, 1 embryo per well in $1.5 \mathrm{ml}$ of egg water. Behavior was recorded between 4 and $6 \mathrm{dpf}$ using a Zebrabox imaging system (Viewpoint Life Sciences) and tracked with automated video-tracking software (Zebralab; sion at each individual time point. grin $1 b$

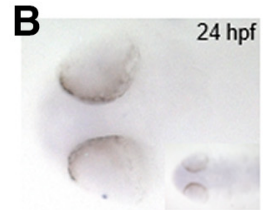

D

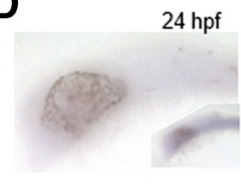

$24 \mathrm{hpf}$

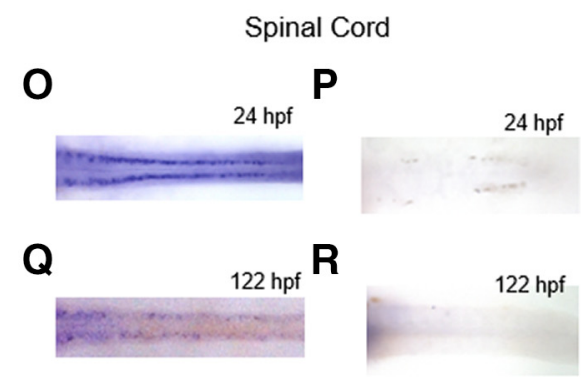

S

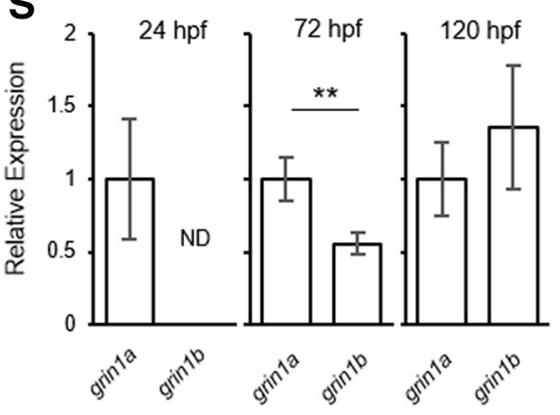

M
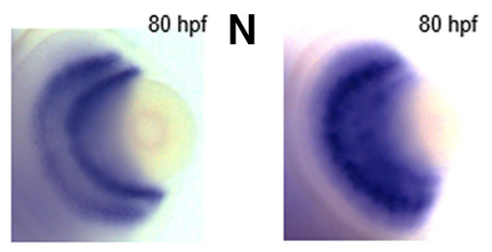

Figure 2. Expression of grin1a and grin1b in the zebrafish nervous system. Whole-mount ISH of grin1a and grin1b at $24 \mathrm{hpf}(\boldsymbol{A}-\boldsymbol{D}), 80 \mathrm{hpf}(\boldsymbol{E}-\boldsymbol{H})$, and $122 \mathrm{hpf}(\boldsymbol{I}-\boldsymbol{L})$ showing dorsal $(\boldsymbol{A}, \boldsymbol{B}, \boldsymbol{E}, \boldsymbol{F}, \boldsymbol{I}, \boldsymbol{J})$ and lateral $(\boldsymbol{C}, \boldsymbol{D}, \boldsymbol{G}, \boldsymbol{H}, \boldsymbol{K}, \boldsymbol{L})$ views. $\boldsymbol{B}, \boldsymbol{D}, \boldsymbol{F}, \boldsymbol{H}$, paired $t$ test), and $120 \mathrm{hpf}(n=6)$ in WT larvae from qPCR. ND, Not detected. Results are normalized to grin1a expres-

Viewpoint Life Sciences). Gross motor movements were reported as movement count, the number of distinct movements generated, averaged per genotype.

Acoustic startle behavior paradigm. Individual fish were placed in 9well $\sim 1 \mathrm{~cm}^{2}$ gridded plates and tested for changes in short latency startle responses (SLC) to acoustic/vibrational stimuli and habituation of SLC. Pulsed acoustic stimuli were delivered using a Type 3810 minishaker (Bruel-Kjaer) and controlled using a BNC-2110 DAQ board (National Instruments). Behavioral responses were imaged at 1000 frames/s using a DRS Lightning high speed camera (DEL Imaging) and analyzed using FLOTE software (Burgess and Granato, 2007a). SLC responses were assigned based on response latency and kinematics. Control larvae were WT and heterozygous siblings from the same clutch as mutants. We assigned larvae to each group by genotyping after behavioral analysis.

For SLC and PPI experiments, each plate of fish was presented with 20 presentations each of (1) high-intensity startle stimuli ( $37 \pm 1 \mathrm{~dB}$ re. $\left.\mathrm{m} / \mathrm{s}^{2}\right),(2)$ low-intensity startle stimuli $\left(19 \pm 1 \mathrm{~dB} \mathrm{re} . \mathrm{m} / \mathrm{s}^{2}\right)$, and prepulse stimuli of $19 \pm 1 \mathrm{~dB} \mathrm{~dB}$ re. $\mathrm{m} / \mathrm{s}^{2}$ followed by high-intensity startle stimuli at (3) $50 \mathrm{~ms}$ ISI and (4) $500 \mathrm{~ms}$ ISI. These 80 stimuli were delivered in 


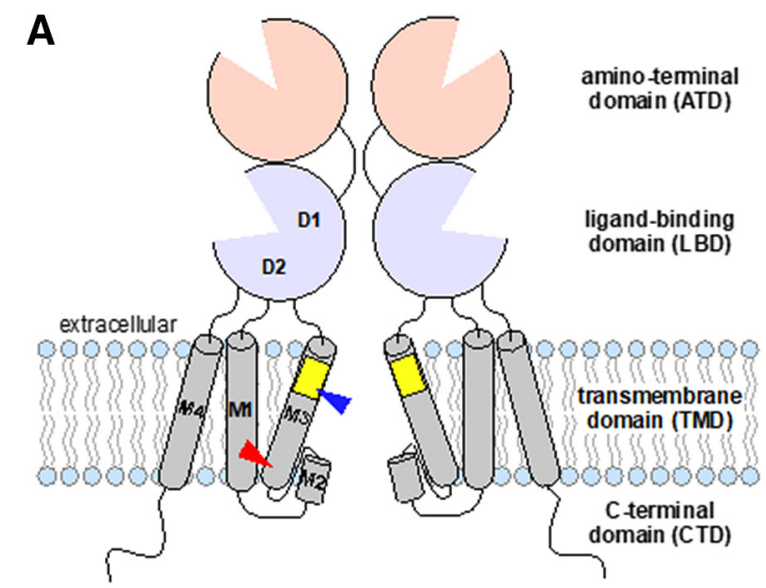

B

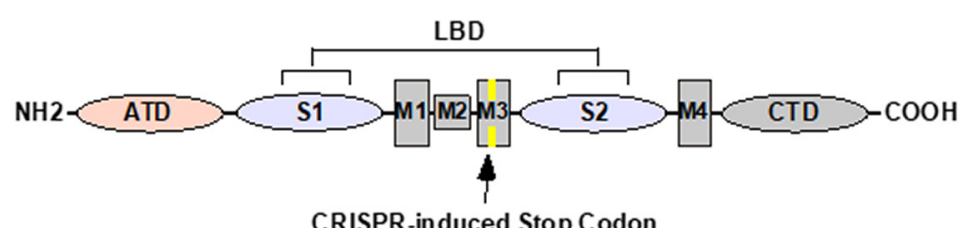

C

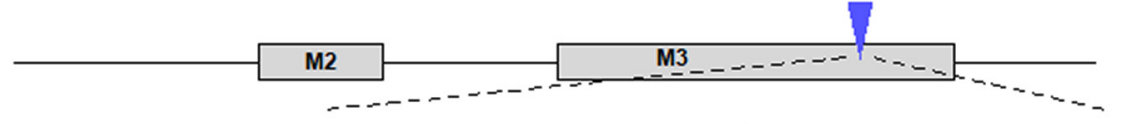

grinla wildtype grinla sbugo $(\Delta 7)$ grinla sbug1 $(\Delta 5+9)$ grinia sbu92 $(\Delta 4)$
ATTATAGTAGCATCCTATACTGCCAACCTGGCTGCCTTCCTAGTGTTGGACCGGCC ATTATAGTAGCATCCTATAC-------CTGGCTGCCTTCCTAGTGTTGGACCGGCC

ATTATAGTAGCATCCTATACCCCCCAGGC-----ACCTGGCTGCCTTCCTAGTGTTGGACCGGCC ATTATAGTAGCATCCTATACT----ACCTGGCTGCCTTCCTAGTGTTGGACCGGCC

\section{grinla wildtype \\ grinla sbugo( $\Delta 7)$ \\ SFSARILGMVWAGFAMIIVASYTANLAAFLVLDRP \\ grinla sbug1 $(\Delta 5+9)$ \\ SFSARILGMVWAGFAMI IVASYTWLPSSTOP \\ grinla sbug2 $(\Delta 4)$ \\ SFSARILGMVWAGFAMI IVASYT PQAPGCLPSVGPASTOP \\ SFSARI LGMVWAGFAMI IVASYTTWLPSSTOP}

\section{D}

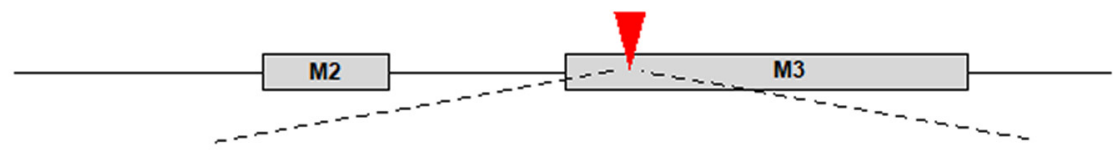

grin1b wildtype grin1b sbu93 $(\Delta 5)$ grin1b sbu94 $(+17)$ grin1b sbug5 $(\Delta 5)$

grinib wildtype grin1b sbu93 $(\Delta 5)$ grin1b sbug4 $(+17)$ grin1b sbu95 $(\Delta 5)$
AGCTTTTCGGCGAGGATTTTAGGTATGGTGTGGGCTGGTTTTGCTATGATTATAG AGCTTTTCGGCGAGGATTTTAGGT-----GTGGGCTGGTTTTGCTATGATTATAG AGCTTTTCGGCGAGGATTTTAGGTATGGATTTTAGGTATGGTACATGTGGGCTGGTTTTGCTATGATTATAG AGCTTTTCGGCGAGGATTTTAGGTA-----TGGGCTGGTTTTGCTATGATTATAG
E

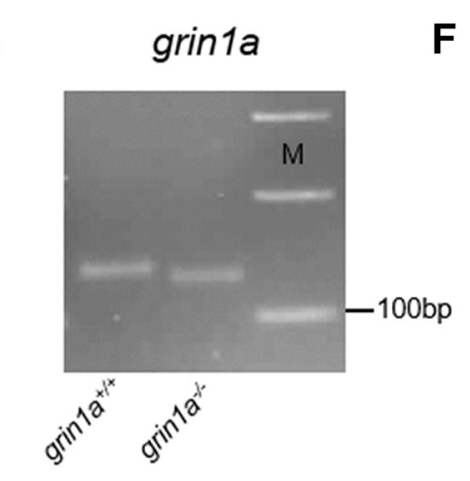

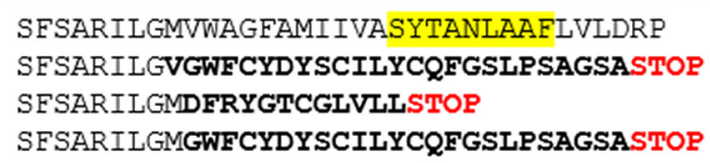

$\mathbf{F}$

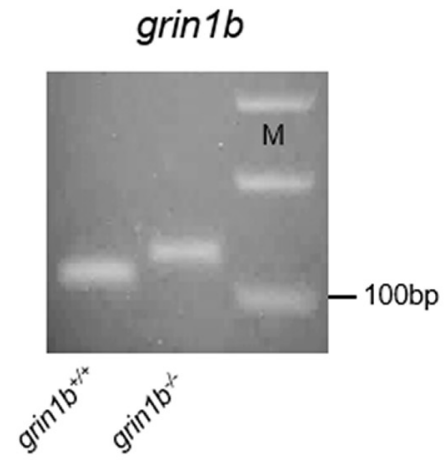

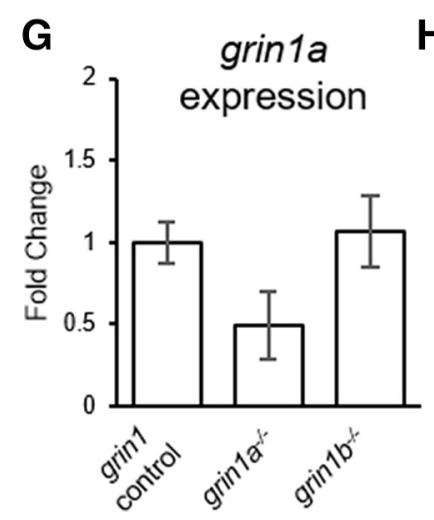

Figure 3. Generation of loss-of-function lesions in grin1a and grin1b using CRISPR-Cas9. A, Schematic depiction of membrane topology of two NMDAR subunits (functional NMDARs are tetramers). Blue and red arrows indicate approximate sites for gRNA targets for grin1a and grin1b, respectively. NMDARs are composed of four modular domains: the extracellular ATD and LBD; the membrane-embedded TMD; and the intracellular CTD. Each individual subunit contributes three transmembrane segments (M1, M3, and M4) and an M2 pore loop to form the ion channel. The most highly conserved motif in iGluRs, the SYTANLAAF motif (labeled in yellow), is within the M3 segment. gRNAs were designed to prevent generation of this motif and downstream elements. $\boldsymbol{B}$, Linear representation of the NMDAR subunit protein sequence, highlighting elements downstream of the gRNA lesion sites in the grin1 mutants that are predicted to be absent, including half of the LBD (S2) and all of the M4 and CTD. In the linear representation, the two lobes of the LBD are represented as S1 and S2; whereas in the 3D representation of the structure, 
pseudorandom order with a $30 \mathrm{~s}$ interval between consecutive stimulus presentations. PPI was calculated as the percent decrease in SLC responsiveness in the prepulse trials compared with no prepulse trials. To control for baseline SLC responsiveness, PPI analysis was only conducted on fish that responded in $30 \%-95 \%$ of trials in the high-intensity startle stimulus alone condition.

For habituation experiments, larvae were presented with high-intensity startle stimuli with $1 \mathrm{~s}$ interval between stimulus presentations. This paradigm has demonstrated rapid habituation, with SLC decreases of up to $80 \%$ after 20 stimulus presentations (Wolman et al., 2011). Because of the stochasticity of individual-level data, we analyzed responsiveness in 4 epochs of 10 stimuli. Habituation was quantified as the percent decrease in SLC responsiveness in the last epoch relative to the first epoch.

Experimental design and statistical analysis

Data analysis was performed using IgorPro, Excel, and MiniTab 18. All average values are presented as mean \pm SEM. The number of replicates is indicated in the figure legends. In instances where we were only interested in whether outcomes were statistically different from WT or an appropriate control, we used an unpaired two-tailed Student's $t$ test to test for significance. In instances where we were interested in how multiple groups varied from each other, we used a one-factor ANOVA and followed with a post hoc Tukey's test. Statistical significance was set at $p<0.05$.

Sex in zebrafish is indeterminate at the age of our larval studies and was thus not a factor in our experimental design.

\section{Results}

Functional NMDARs are heterotetramers composed of two obligatory GluN1 subunits and typically some combination of GluN2 (A-D) subunits. Initially, we studied the functional properties of the zebrafish grin1 (GluN1) paralogs, grin1a and grin $1 b$.

\section{NMDARs containing zebrafish GluN1 paralogs are highly $\mathrm{Ca}^{2+}$-permeable}

NMDARs are ligand-gated ion channels (Paoletti et al., 2013). To define potential functional differences between zebrafish paralogs, we expressed grin1a and grin1b in a heterologous expression system (see Materials and Methods). To reduce potential variations with the GluN2 subunit, transfected constructs to express fish GluN1a (zGluN1a) or GluN1b (zGluN1b) with rat GluN2A (rGluN2A) (Fig. 1). As a reference, we compared these recordings with those for rat GluN1 (rGluN1) coexpressed with rGluN2A (rGluN1/rGluN2A).rGluN1/rGluN2A

they are designated D1 (mainly composed of S1) and D2 (mainly composed of S2). C, D, Schematic of gRNA target sites for grin $1 a$ (blue arrow) $(\boldsymbol{C}$ and grin $1 b$ (red arrow) $(\boldsymbol{D})$ as well as alignments of nucleotide (top) and amino acid (bottom) sequences. Induced mutations within the nucleotide sequences are denoted as either dashes (deletions) or highlighted blue (insertions). gRNA target site on the nucleotide sequence (gray highlight) and PAM sites (bolded) are adjacent to generated mutations. Altered amino acid sequence (bolded) and early stop codons (red STOP) are generated in all alleles, disrupting the SYTANLAAF motif (yellow highlight) as well as removing the D2 (S2) lobe of the LBD, which would make the receptor nonfunctional. $\boldsymbol{E}, \boldsymbol{F}$, Lesions altered $\mathrm{mRNA}$ size in expected fashions. CDNA amplification of the following: $(\boldsymbol{E})$ grin $1 a^{+/+}$and grin $1 a^{-/-}$(unless otherwise noted, grin $1 a^{-1-}$ indicates the sbu90 allele) producing expected product sizes of 147 and $140 \mathrm{bp}$, respectively; and $(\boldsymbol{F})$ grin $1 b^{+/+}$and grin $1 b^{-1-}$ (unless otherwise noted, grin $1 b^{-/-}$indicates the sbu94 allele) producing expected product sizes of 109 and $126 \mathrm{bp}$, respectively. M, Marker. For all genotypes, RNA was collected from homozygous intercrosses at $3 \mathrm{dpf}$. $\boldsymbol{G}, \boldsymbol{H}, \mathrm{qPCR}$ of grin1a (G) and grin $1 b(\boldsymbol{H})$ expression at $5 \mathrm{dpf}$ in grin1 control $(n=6)$, grin1a ${ }^{-1-}(n=6)$, and grin $1 b^{-1-}(n=6)$ fish. $\mathbf{G}, \boldsymbol{H}$, None of the values was significantly different.
A

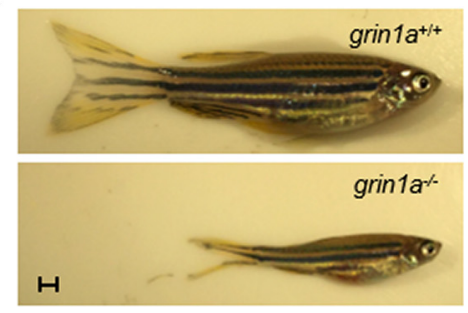

B

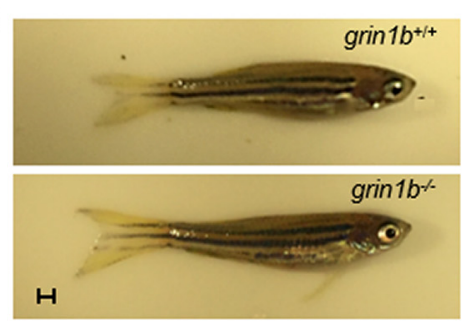

C
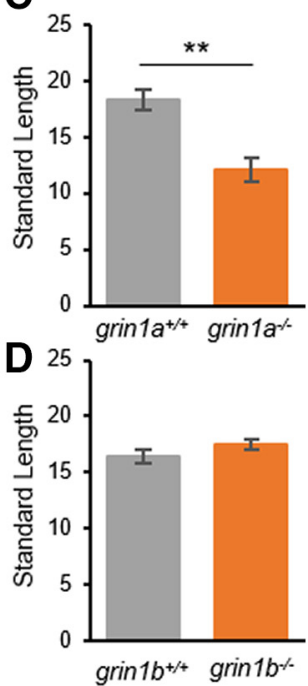

E

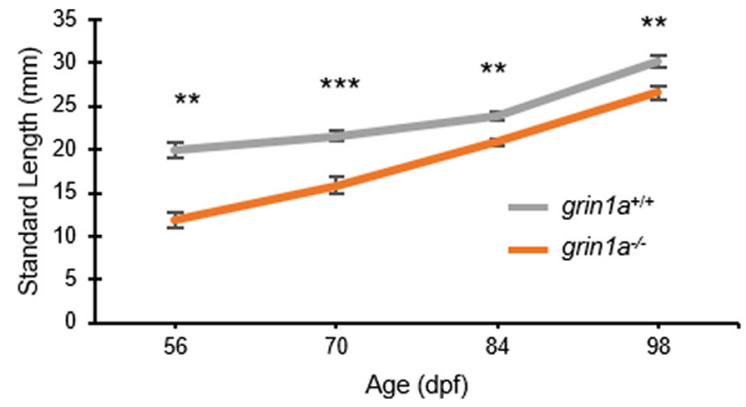

Figure 4. grin $1 a^{-1-}$ but not grin $1 b^{-1-}$, fish have decreased growth into adulthood. $\boldsymbol{A}$, $\boldsymbol{B}$, Representative photographs of grin $1 a^{+1+}$ and grin $1 a^{-1-}$ fish $(\boldsymbol{A})$ or of grin $1 b^{+1+}$ and grin $1 b^{-1-}$ fish $(\boldsymbol{B})$ at 2 months after fertilization. Scale bars, $1 \mathrm{~mm}$. $\boldsymbol{C}, \boldsymbol{D}$, Standard length comparison (mean \pm SEM) of $(C)$ grin $1 a^{+/+}(n=17)$ and grin $1 a^{-1-}(n=5)$ $\left({ }^{* *} p=0.0014, t\right.$ test $)$ or $(\boldsymbol{D})$ of grin $1 b^{+/+}(n=17)$ and grin $1 b^{-1-}(n=31)$ at 2 months after fertilization. As different conditions during development may contribute to size variations between crosses (see grin $1 a^{+/+}[\boldsymbol{A}]$ vs grin $1 b^{+/+}[\boldsymbol{B}]$ controls), we only made size comparisons between within-cross controls. $\boldsymbol{E}$, Standard length comparison from 8 weeks (56 dpf, ${ }^{* *} p=0.0014$, $t$ test), 10 weeks (70 dpf, ${ }^{* * *} p=6.5 \mathrm{e}-4, t$ test), 12 weeks (84 dpf, ${ }^{* *} p=0.0054, t$ test), and 14 weeks (98 dpf, ${ }^{* *} p=0.0067, t$ test) for grin $1 a^{+1+}(n=17)$ and grin $1 a^{-1-}(n=5)$. After initial measurement at 8 weeks, fish were moved to individual tanks.

shows strong expression in HEK293 cells (Alsaloum et al., 2016; Amin et al., 2017) (Fig. 1A, left). NMDARs containing zGluN1a (Fig. $1 A$, middle) or zGluN1b (Fig. $1 A$, right) also showed robust current amplitudes that were indistinguishable from rGluN1 (Fig. 1B). Hence, both zGluN1a and zGluN1b form functional channels with rat GluN2A subunits.

A critical physiological property of NMDARs is their high $\mathrm{Ca}^{2+}$ permeability (Paoletti et al., 2013), which is dominated by GluN1 (Burnashev et al., 1992; Watanabe et al., 2002). We therefore assayed the $\mathrm{Ca}^{2+}$ permeability $\left(\mathrm{P}_{\mathrm{Ca}}\right)$, relative to $\mathrm{Na}^{+}\left(\mathrm{P}_{\mathrm{Ca}} /\right.$ $\mathrm{P}_{\mathrm{Na}}$ ), of NMDARs containing zGluN1a or zGluN1b (Fig. 1C,D). To measure $\mathrm{P}_{\mathrm{Ca}} / \mathrm{P}_{\mathrm{Na}}$, we measured changes in reversal potentials going from a high $\mathrm{Na}^{+}$solution without $\mathrm{Ca}^{2+}$ to the same solution containing $10 \mathrm{mM} \mathrm{Ca}^{2+}$ (see Materials and Methods) (Jatzke et al., 2002; Amin et al., 2018). NMDARs containing zGluN1a or zGluN1b showed a high $\mathrm{Ca}^{2+}$ permeability that is indistinguishable from that of NMDARs containing rGluN1 (Fig. 1D). Thus, zebrafish NMDARs, regardless of the GluN1 paralog, are highly $\mathrm{Ca}^{2+}$-permeable, like their mammalian counterpart (Wollmuth, 2018). 
A

\begin{tabular}{|c|c|c|c|c|c|}
\hline Allele & $\begin{array}{c}\text { Age } \\
\text { Screened }\end{array}$ & $\begin{array}{c}\text { Number } \\
\text { Screened }\end{array}$ & $\begin{array}{c}\text { Expected } \\
\text { Percentage }\end{array}$ & $\begin{array}{c}\text { Percentage } \\
\text { Wildtype }\end{array}$ & $\begin{array}{c}\text { Percentage } \\
\text { Mutant }\end{array}$ \\
\hline \multirow{2}{*}{ grin1 double } & $6 \mathrm{dpf}$ & 557 & $6.25 \%$ & $5.2 \%$ & $6.3 \%$ \\
\cline { 2 - 6 } & Adult & 117 & $6.25 \%$ & $7.7 \%$ & $0.0 \%$ \\
\hline
\end{tabular}

B

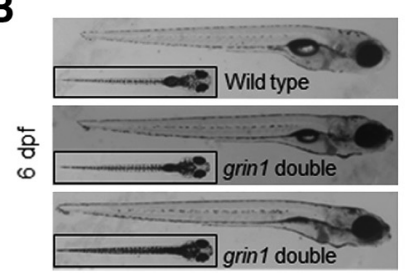

C

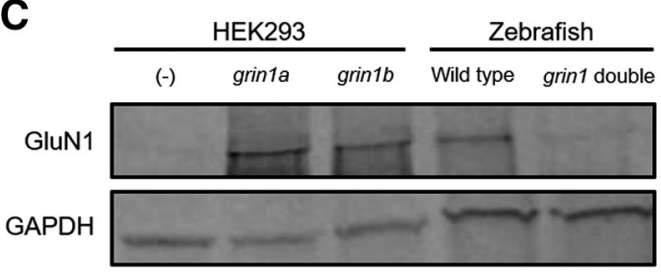

Figure 5. grin1 double mutants can survive through early larvae development. $A$, Viability of grin1 double mutants at $6 \mathrm{dpf}$ and $>2$ months after fertilization generated from double heterozygous intercrosses using the sbu90 and sbu94 alleles for grin $1 a$ and grin1b, respectively. Expected recovery of grin1 double mutants is $1 / 16(6.25 \%)$. B , Lateral views of live images of WT (top) and grin 1 double mutants (middle, bottom) at $6 \mathrm{dpf}$. grin1 double mutants often exhibited uninflated swim bladders (bottom). C, Western blot analysis of GluN1 from HEK293 cells expressing zGluN1a (lane 2) or zGluN1b (lane 3) (along with rat GluN2A), or from zebrafish lysate from WT (lane 4) or grin1 double mutant (lane 5) larvae. GAPDH was used a protein reference.

\section{Differential expression of zebrafish GRIN1 paralogs in early development}

grinla is expressed in the brain, retina, and spinal cord at $1 \mathrm{dpf}$ with expression becoming more robust by $2 \mathrm{dpf}$ (Cox et al., 2005). Over the same period, grin $1 b$ expression is much weaker (Cox et al., 2005). To further define their expression, we studied grin $1 a$ and grin $1 b$ expression using whole-mount RNA ISH over a wider developmental range (1, 3, and $5 \mathrm{dpf}$ ) (Fig. 2). For these experiments, we used the published grin1a probe (Cox et al., 2005 ) but redesigned the grin $1 b$ probe to reduce background (see Materials and Methods).

Consistent with earlier results, we observed expression of grinla at $24 \mathrm{hpf}$ in the telencephalon (Fig. $2 A, C$, arrows) and in the spinal cord (Fig. 2O). We could not detect any grin $1 b$ transcripts at this developmental stage (Fig. $2 B, D, P$ ). By $80 \mathrm{hpf}$, grin $1 a$ and grin $1 b$ are expressed more broadly throughout the brain and in distinct domains in the retina (grin1a: Fig. 2E,G,M; grin $1 b$ : Fig. $2 F, H, N)$. At $122 \mathrm{hpf}$, grin $1 a$ and grin $1 b$ are expressed in the telencephalon, optic tectum, hindbrain (grin1a: Fig. 2I,K; grin $1 b$, Fig. $2 J, L)$ and in the retina (data not shown).

Although both grin1a and grin1b have overlapping expression, there are notable differences. In the spinal cord, grin1a is robustly expressed between 24 and $122 \mathrm{hpf}$, but we could not detect any grin $1 b$ expression at these stages (Fig. 2O-R). In the telencephalon and optic tectum, grin1a is broadly expressed at $122 \mathrm{hpf}$, whereas grin $1 b$ has a more discrete expression pattern (grin1a: Fig. 2I,K; grin1b; Fig. 2J,L).

The RNA ISH experiments suggest that grin1a is expressed earlier and at higher levels than grin1b. To directly test this idea, we conducted qPCR (Fig. $2 S$ ). As expected, grin1a was readily detected at $24 \mathrm{hpf}$, whereas grin $1 b$ could not be detected (Fig. $2 S$, left). In addition, at $72 \mathrm{hpf}$, the expression of grinla was significantly greater than that of grin $1 b$ (Fig. $2 S$, middle), but by 120 hpf, expression levels were comparable (Fig. $2 S$, right). Hence, grin $1 a$ is expressed earlier than grin $1 b$ and shows a more robust expression pattern through $3 \mathrm{dpf}$.

\section{Generation of grin $1 a$ and grin $1 b$ mutations}

To study the requirements for NMDAR-mediated transmission in the developing zebrafish nervous system, we disrupted the grin1 $a$ and grin $1 b$ genes using CRISPRCas9 (Chang et al., 2013; Hwang et al., 2013). We targeted the highly conserved SYTANLAAF motif within the M3 transmembrane segment (Fig. $3 A$ (Wollmuth and Sobolevsky, 2004; Traynelis et al., 2010). Frameshift mutations in this region will prevent formation of the ion channel, the ligand-binding domain (LBD), the last transmembrane domain (M4), and the C-terminal domain (CTD) (Fig. 3B). Any protein generated from these alleles would be unable to bind ligand, and iGluRs lacking the M4 segment do not tetramerize nor reach the membrane (Salussolia et al., 2011, 2013; Gan et al., 2015). We analyzed three germline mutations for each paralog. Sequencing of each lesion revealed a predicted frameshift mutation and a nearby stop codon (Fig. 3C, $D)$. For the majority of our subsequent analysis, we used alleles of grin1a and grin $1 b$ that yielded the earliest predicted stop codon: a 7-nucleotide deletion in grin1a $\left(\right.$ grin $\left.1 a^{\text {sbu90 }}\right)$ and a 17-nucleotide insertion in grin $1 b\left(\right.$ grin $\left.1 b^{\text {sbu94 }}\right)$. RT-PCR only revealed grin1a or grin1b mRNA species containing the indel in the respective mutants (Fig. 3E,F). The M3 segment including the SYTANLAAF is integral to iGluR function; any disruption of the M3 segment would by itself ablate receptor function (Kazi et al., 2014; Hansen et al., 2018). Given the functional importance of the M3 segment, it is improbable that any cryptic splicing event that bypassed the lesion site in this region could produce a functional receptor. Based on the anticipated consequences of the mutations on NMDAR structure, any protein produced from the grinla and grin $1 b$ mutant alleles would not form a functional receptor.

Nonsense-mediated decay of mutant mRNA can cause transcriptional adaptation and upregulation of related genes (ElBrolosy et al., 2019). We therefore assessed levels of the lesioned mRNA and the corresponding paralog in grin $1 a^{-/-}$and grin $1 b^{-1-}$ fish at $5 \mathrm{dpf}$ (Fig. $3 G, H)$. We detected a trending, but not significant, decrease in grin1a expression in grinla ${ }^{-1-}$ larvae at 5 dpf ( $p=0.06, t$ test; Fig. $3 G)$, suggesting possible non-sensemediated decay. However, for grin $1 b^{-1-}$ larvae, we detected no corresponding difference in grin $1 b$ expression (Fig. $3 H$ ). To determine whether transcriptional adaptation enhanced expression of the corresponding paralog, we assayed the expression levels of grin1b in grinla $a^{-1-}$ (Fig. $3 H$ ) and of grinla in grin $1 b^{-1-}$ (Fig. $3 G$ ) at $5 \mathrm{dpf}$ but did not detect compensation. grin $1 a$, but not grin $1 b$, is expressed in the spinal cord at $5 \mathrm{dpf}$ (Fig. 2Q,R). We therefore also assayed grin $1 b$ expression in the spinal cord of $5 \mathrm{dpf}$ grin $1 a^{-l-}$ larvae by RNA ISH of intact larvae and by qPCR of spinalized larvae. We did not detect compensation by either method (data not shown). Together, transcriptional upregulation of the corresponding paralog is not a prominent feature of the grin1 alleles at larval stages.

\section{grin $1 a$ and grin $1 b$ single-mutant fish are viable, but grin1a mutants display growth defects}

grin $1 a$ and grin $1 b$ single-mutant fish appear morphologically normal at $6 \mathrm{dpf}$. Although both survive to adulthood and are fertile, grin $1 a^{-1-}$ adults are not recovered at expected Mendelian ratios. Of the 292 adult fish generated from grin $1 a^{+/-}$ 
intercrosses, only 35 were grin $1 a^{-/-}$ fish, resulting in $11.9 \%$ recovery instead of the predicted $25.0 \%\left(p=1.6 \mathrm{e}-6, \chi^{2}\right.$ test). Adult grin $1 a^{-1-}$ fish that are recovered are smaller than their WT siblings (Fig. $4 A, C$ ). In contrast, grin $1 b^{-l-}$ fish show no growth nor viability deficits (Fig. 4B,D). For grin $1 a^{-1-}$ fish, a size deficit compared with their WT siblings is apparent between 56 and $98 \mathrm{dpf}$, although this narrows later in development (Fig. 4E). The same reduced viability and growth deficit observed in grin $1 a^{\text {sbu } 90 / \text { sbu } 90}$ was also present in a second allele, grin1a sbu92 (data not shown). These phenotypic

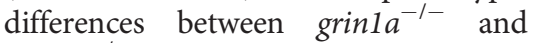
grin $1 b^{-/-}$fish indicate that GluN1b is unable to fully compensate for the loss of GluN1a in grin1 $1 a^{-1-}$.

\section{Zebrafish lacking grin1 survive until 10 dpf}

Knockout of grin 1 in mice is embryonic lethal (Forrest et al., 1994). Both grin1a and grin $1 b$ single-mutant fish survive to adulthood and produce viable offspring. Zebrafish grin1 double mutants $\left(\right.$ grin $1 a^{-1-} ;$ grin $\left.1 b^{-1-}\right)$ are recovered at expected Mendelian ratios at $6 \mathrm{dpf}$ (Fig. $5 A$ ) but do not survive past $10 \mathrm{dpf}$ (Fig. 5A; and data not shown). The majority of grin1 double mutants fail to fully inflate their swim bladders (Fig. $5 B$ ) but grow at comparable rates through early larval development (data not shown).

To confirm the absence of GluN1 in the grin 1 double mutants, we performed Western blot analysis (Fig. 5C). As a control, we extracted lysates from HEK293 cells expressing either grin1a or grin1b, coexpressed with rat GluN2A. The GluN1 antibody binds to both zGluN1a and zGluN1b (Fig. 5C, lanes 2 and 3) and showed a band of the expected size in protein lysates extracted from the heads of $7 \mathrm{dpf}$ WT fish (Fig. $5 C$, lane 4). In contrast, we were unable to detect GluN1 immunoreactivity in $7 \mathrm{dpf}$ grin 1 doublemutant larvae (Fig. 5C, lane 5). These results are consistent with our RT-PCR data (Fig. 3D,E), indicating an absence of WT grin1 transcripts and protein in mutant fish.

\section{NMDARs are required for normal feeding behavior}

Fish lacking NMDARs can survive until $10 \mathrm{dpf}$ (Fig. 5). Given the importance of NMDARs to brain function, we wanted to understand the complexity of behaviors that the grin 1 doublemutant larvae could generate. To begin to address this question, we assayed prey-capture, which is a complex behavior requiring visual and motor coordination (Semmelhack et al., 2014) and is an essential behavior for survival. We used a larval feeding assay to assess the ability of individual larvae to capture paramecia (Fig. 6A) (Gahtan et al., 2005). Because of variation in the number of paramecia eaten by control larvae from day to day, we normalized the proportion of paramecia eaten by the experimental group to their same-day control (Fig. $6 B-D$ ).
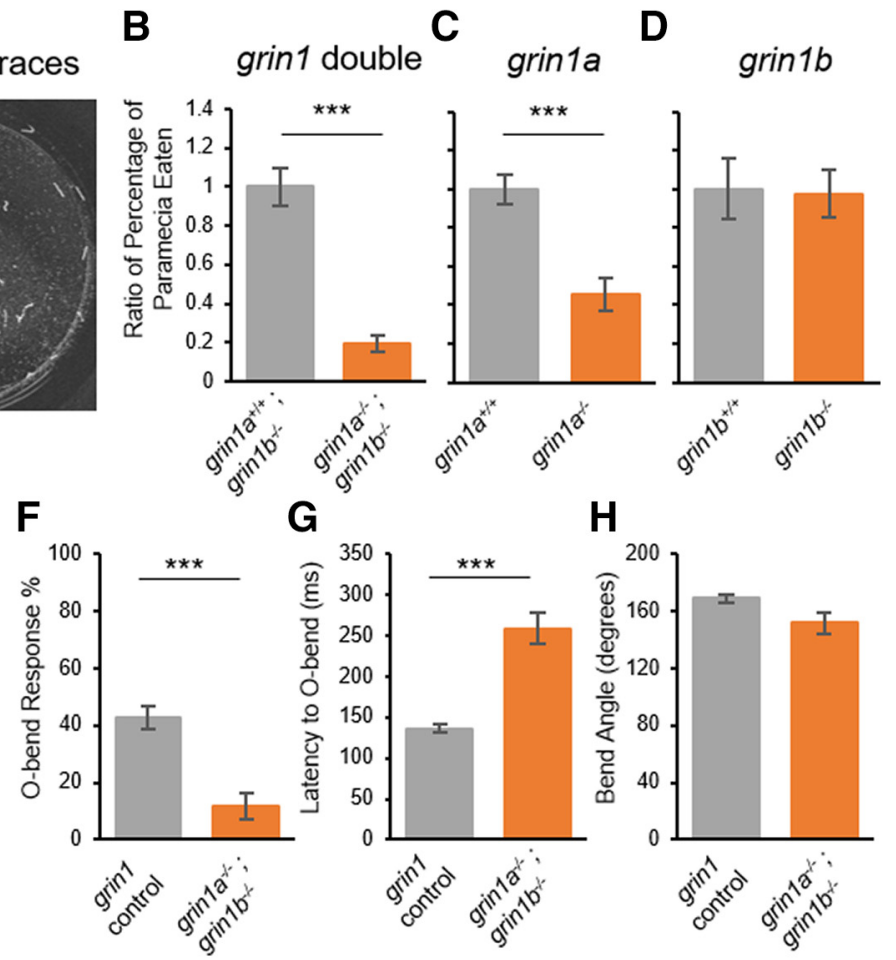

Figure 6. grin1 mutations cause feeding and visually evoked startle deficits. $\boldsymbol{A}$, Representative traces of paramecium movement used to assay feeding. Traces generated by analyzing $2.5 \mathrm{~s}$ of paramecia movement. $\boldsymbol{B}-\boldsymbol{D}$, Proportion of paramecia eaten over the trial period (mean \pm SEM) normalized to control for the following: $(\boldsymbol{B})$ grin1 control (grin $\left.1 a^{+/+} ; \operatorname{grin} 1 b^{-1-}, n=18\right)$ or $\operatorname{grin1}^{-1-} ; \operatorname{grin}^{-1-}(n=33)$ fish $\left(^{* * *} p=1.8 \mathrm{e}-11, t\right.$ test $) ;(C)$ grin1a ${ }^{+/+}(n=25)$ or grin $1 a^{-1-}(n=23)$ fish $\left({ }^{* * *} p=8.9 \mathrm{e}-6, t\right.$ test $)$; and $(\boldsymbol{D})$ grin $1 b^{+/+}(n=14)$ or grin $1 b^{-1-}(n=10)$ fish. $\boldsymbol{E}$, Representative response to dark flash stimulus from resting (EI) to characteristic 0 -bend (Eiii) and reorientation (Eiv) of a WT zebrafish larva. $\boldsymbol{F}$, Percentage of larvae bend response ( ${ }^{* *} p=4.6 \mathrm{e}-13, t$ test); and $(\boldsymbol{H})$ bend angle of response for 0 -bends $(n=104, n=14)$ generated by grin 1 control (grin $1 a^{+/+} ;$grin $1 b^{-/-}$or grin $1 a^{+/-} ;$grin $\left.1 b^{-/-}\right)$or grin $1 a^{-/-} ;$grin $1 b^{-/-}$, respectively.

Compared with their controls, grin1 double-mutant larvae showed a significant deficit in prey-capture (Fig. 6B). Nevertheless, these fish could successfully perform this complex behavior. We also tested the grin 1 single mutants in this assay. Like the grin1 double mutant, grin $1 a^{-1-}$ also showed a significant deficit in prey-capture (Fig. 6C), a phenotype recapitulated in the grin $1 a^{\text {sbu92 }}$ allele (data not shown). No deficits were observed in grin $1 b^{-1-}$ larvae (Fig. 6D). Hence, the grin1 double-mutant and grin $1 a^{-1-}$ fish show a notable deficit in prey-capture. This inability to efficiently capture prey may contribute to the early death of grin 1 double mutants (Fig. 5) and reduced growth rate of grin 1a single mutants (Fig. 4).

\section{Loss of NMDAR results in visual deficits}

Zebrafish are highly visual animals (Gestri et al., 2012). One possible explanation for the reduced prey-capture is a disruption in vision. Indeed, both grin 1 paralogs are expressed in the retina (Fig. 2M,N) and NMDAR-mediated transmission is necessary for proper development of the visual system (Schmidt et al., 2000). To assay vision, we used a stereotyped behavioral response, the O-bend, which is a visually evoked response to a dark flash stimulus (Fig. 6E) (Burgess and Granato, 2007a; Wolman et al., 2011).

Compared with its sibling grin 1 controls, grin 1 double-mutant fish showed a significant decrease in the number of O-bends 
A

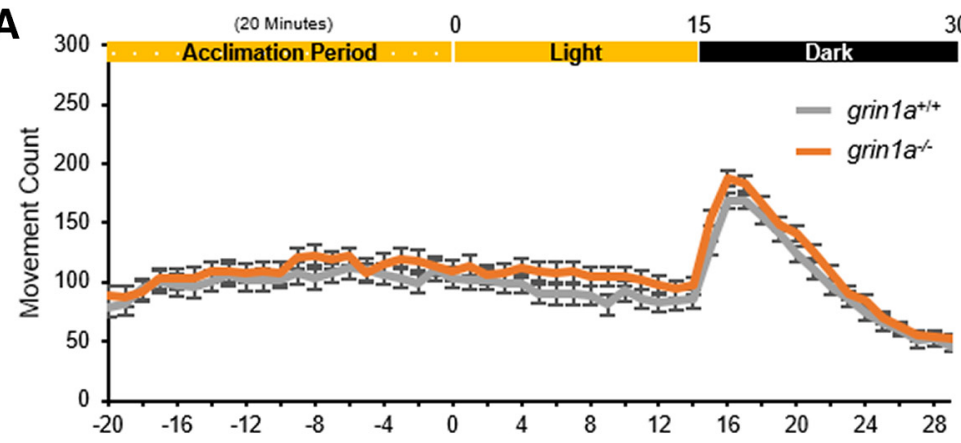

B

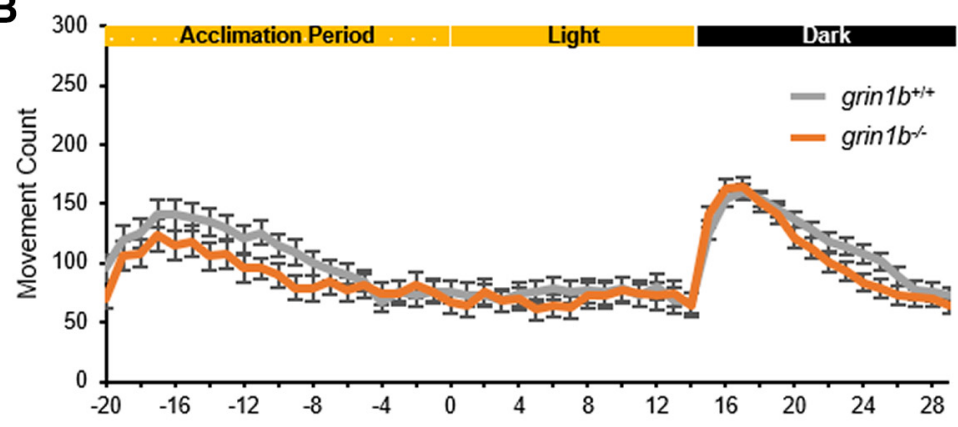

C

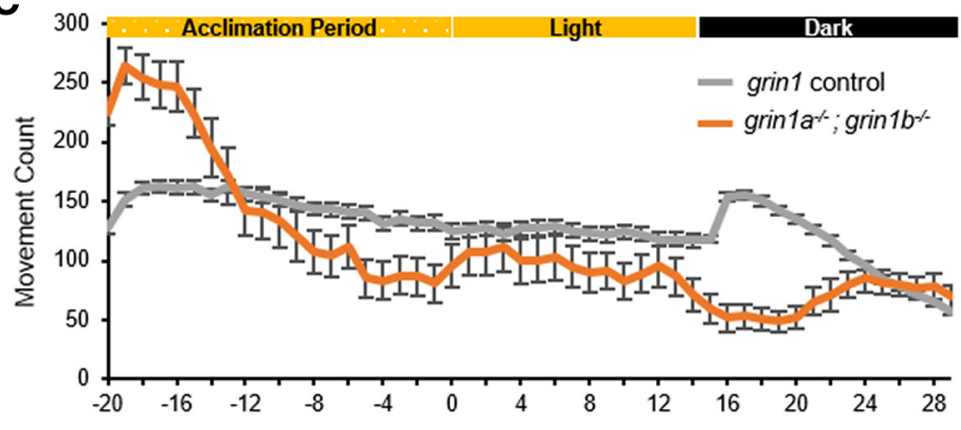

D

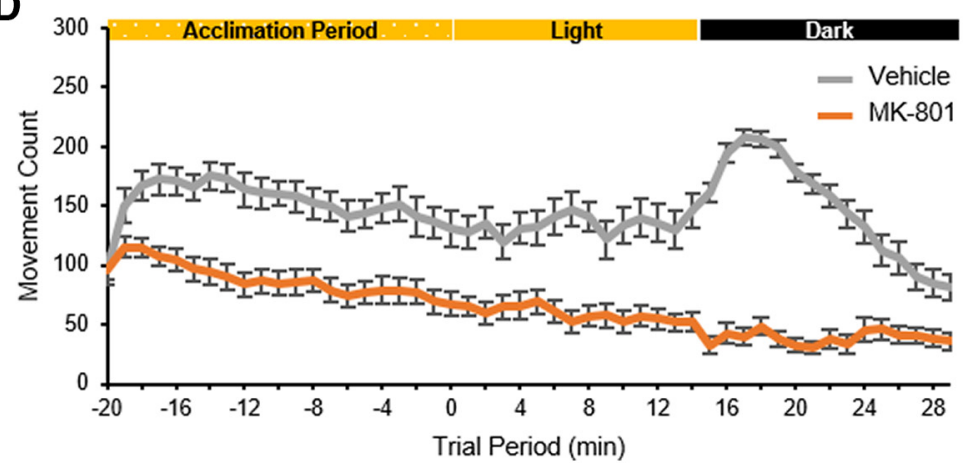

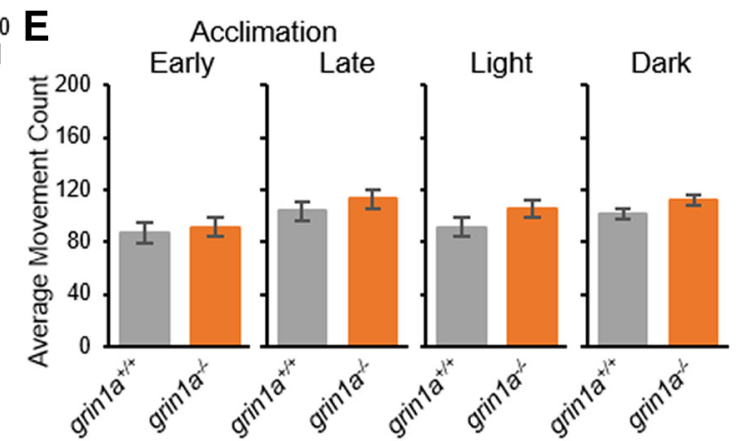

F Acclimation

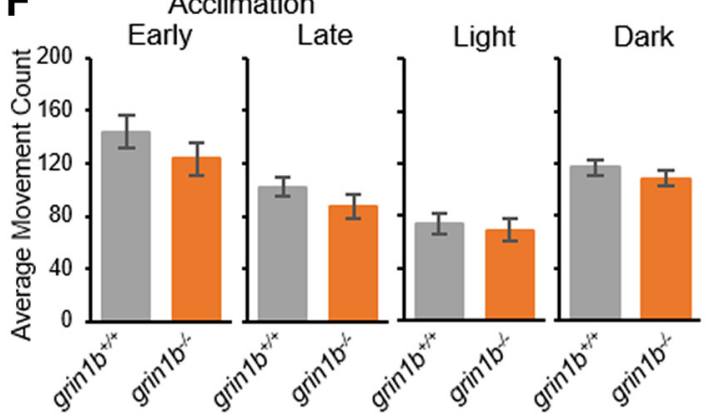

G

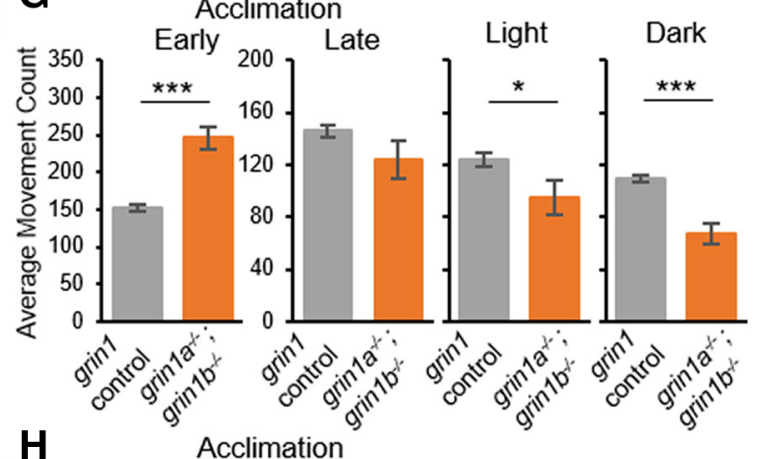

H

Acclimation

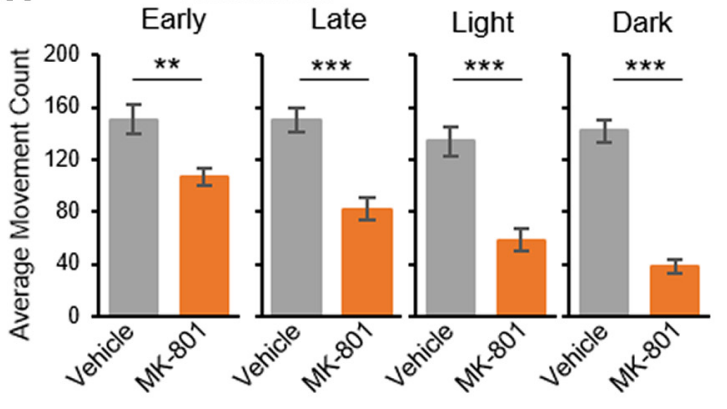

Figure 7. The grin 1 double mutant and MK-801-treated, but not grin $1 a^{-1-}$ or grin $1 b^{-1-}$, alter spontaneous movement. $\mathbf{A}-\mathbf{D}$, Spontaneous movements at 6 dpf and evoked movements after a transition from light to dark. Line graphs of average number of movement counts per minute (mean \pm SEM) for the following: $(\boldsymbol{A}) \mathrm{grin}^{+/ /+}(n=64)$ or grin $1 a^{-/-}(n=79)$ fish; $(\boldsymbol{B})$ $\operatorname{grin}_{1} b^{+/+}(n=49)$ or grin $1 b^{-1-}(n=50)$ fish; $(C)$ grin1 control $\left(\operatorname{grin} 1 a^{+/+} ; \operatorname{grin} 1 b^{+/+},{\operatorname{grin} 1 a^{+/+}}_{\text {grin } 1 b^{+/-}}\right.$and grin $1 a^{+/-} ;$grin $\left.1 b^{+/+}, n=148\right)$ or grin1a $a^{-1-} ;$ grin $1 b^{-1-}(n=32)$; and (D) fish treated with vehicle (0.1\% DMSO) $(n=26)$ or the NMDAR antagonist MK-801 (20 $\mu$ M in 0.1\% DMSO) $(n=27)$. Vehicle or MK-801 was acutely administered $1 \mathrm{~h}$ before the start of the spontaneous movement trial and was included in the bath solution throughout the trial. $\boldsymbol{E}-\boldsymbol{H}$, Bar graphs, of associated spontaneous movement trials, depicting average movements (mean \pm SEM) during the early acclimation (first $5 \mathrm{~min}$ of acclimation), late acclimation (last $15 \mathrm{~min}$ of acclimation), and light and dark periods for the following: (E) grin $1 a^{+/+}$or grin $1 a^{-1-} ;(\boldsymbol{F})$ grin $1 b^{+/+}$or grin $1 b^{-1-} ;(\boldsymbol{G})$ grin1 control or grin1 double mutant $\left({ }^{* * *} p=1.9 \mathrm{e}-13\right.$ for early acclimation; ${ }^{*} p=0.025$ for light; ${ }^{* * *} p=6.7 \mathrm{e}-10$ for dark, $t$ test); and $(\boldsymbol{H})$ vehicle or MK-801-treated fish $\left({ }^{* *} p=0.0012\right.$ for early acclimation; ${ }^{* * *} p=2.1 \mathrm{e}-6$ for late acclimation; ${ }^{* * *} p=2.6 \mathrm{e}-6$ for light; ${ }^{* * *} p=1.4 \mathrm{e}-14$ for dark, $t$ test).

in response to a dark flash (Fig. 6F). When an O-bend was evoked, their latency to initiation was also significantly slower (Fig. $6 G$ ). Neither the grinla nor grin1b single mutants showed any alteration in O-bend responsiveness or latency (data not shown). Of the O-bends generated by the grin1 double-mutant fish, there were no changes in the kinematic parameters of bend angle (Fig. $6 \mathrm{H}$ ) or angular velocity (data not shown), suggesting that the O-bends, although infrequently generated, are normal. These findings suggest the motor circuit underlying this behavior is largely intact. 
In summary, the significantly reduced dark-stimulus evoked response suggests that grin1 double-mutant fish have strongly impaired, but not entirely absent, visual function. In contrast, the visual function of the grin1 single mutants is sufficient to respond normally to dark flash stimuli. Although both grin $1 a$ single and grin 1 double-mutant fish have a prey-capture deficit, only the grin 1 double-mutant fish have a robust visual deficit. Hence, the basis for prey-capture deficits is complex and likely to involve defects outside the visual system.

\section{Spontaneous and evoked movements are disrupted in grin 1 double mutant and MK-801-treated fish}

To further understand the requirement of NMDARs in larval zebrafish behavior, we used a spontaneous and lightto-dark evoked swimming protocol. This protocol provides an overview of motor control and response to a photic stimulus, which is driven by both deep brain photoreceptors and retinal signaling (Fernandes et al., 2012). In addition to assaying locomotor behavior of grin1 mutants, we also tested WT larvae acutely treated with MK801, an NMDAR channel blocker (Traynelis et al., 2010), to begin distinguishing acute and developmental roles of NMDAR-mediated transmission in generating these behaviors. MK-801 is highly specific for NMDARs over non-NMDAR subtypes (Huettner and Bean, 1988) and does not show any preference for different GluN2 subunits (Traynelis et al., 2010).

We monitored spontaneous movements of individual $6 \mathrm{dpf}$ grin $1 a$ and grin $1 b$ single mutants and grin 1 double-mutant larvae in 24-well plates during a 50 min paradigm with periods in light (acclimation and light periods) and dark. Turning off the illumination evokes behavior referred to as a visual motor response (Burgess and Granato, 2007a; Emran et al., 2008). In this paradigm, WT zebrafish display stereotyped behaviors (Fig. $7 A-D$, gray traces): their number of movements stabilizes during the acclimation period and then transiently increases following the transition to darkness. grin $1 a^{-1-}$ (Fig. $\left.7 A, E\right)$ and grin $1 b^{-1-}$ (Fig. $7 B, F)$ fish replicate this behavior. In contrast, although grin 1 double-mutant larvae achieve coordinated swimming, they exhibit a different behavioral profile (Fig. 7C): they are hyperactive during the initial acclimation period, have reduced levels of activity during the light period, and show a decrease in activity on the change to the dark period (Fig. $7 C, G$ ). Other swim parameters in the grin 1 double-mutant larvae, such as duration of movements and distance traveled, showed the same alterations as observed in the movement count (data not shown). These behavioral alterations were not dependent on swim bladder inflation (data not shown).

This altered phenotype in grin1 double-mutant fish could reflect that a behavior requires NMDAR-mediated transmission or that an underlying circuit failed to develop properly in the absence of NMDAR-mediated transmission. To distinguish these possibilities, we applied MK-801, an NMDAR channel blocker, to WT fish $1 \mathrm{~h}$ before behavioral testing (Fig. $7 D$ ) to acutely antagonize NMDAR-mediated transmission. Any phenotype present in the grin 1 double mutants that is recapitulated by MK801 treatment suggests that the phenotype is generated by the lack of NMDAR-mediated transmission, whereas if MK-801 treatment does not replicate the phenotype, it suggests a developmental effect of the long-term absence of NMDAR-mediated signaling. In the spontaneous and photic-evoked movement paradigm, we observed both outcomes. In parallel with the grin1 double-mutant fish, acute MK-801 treatment significantly reduced spontaneous activity during the light period and eliminates the stereotypic response to the light change (Fig. $7 \mathrm{H}$ ). In contrast to the grin1 double-mutant fish, acute MK-801 treatment did not induce hyperactivity during the initial acclimation period (Fig. $7 H$ ).

\section{MK-801 treatment phenocopies multiple behaviors observed in the grin 1 double mutants}

grin1 double mutants both showed decreases in gross movement counts throughout the trial and lacked the stereotypical visual motor response found in WT larvae (Fig. 7G), outcomes phenocopied by MK-801 treatment (Fig. $7 H$ ). Nevertheless, how robust this overlap in behavior is between grin1 double-mutant and MK-801-treated fish is unclear given the gross nature of our movement measurements. We therefore characterized additional swim parameters, as well as the instantaneous response to the transition from light to dark in more detail (Fig. 8). 
A

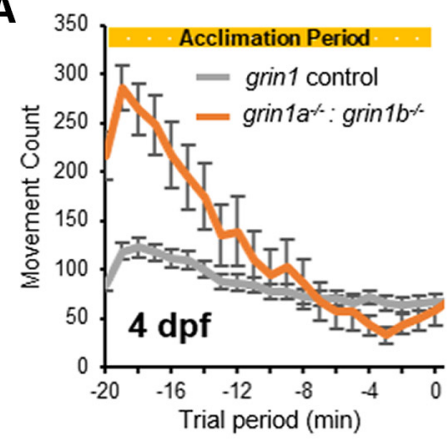

C

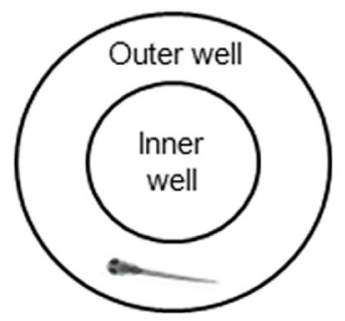

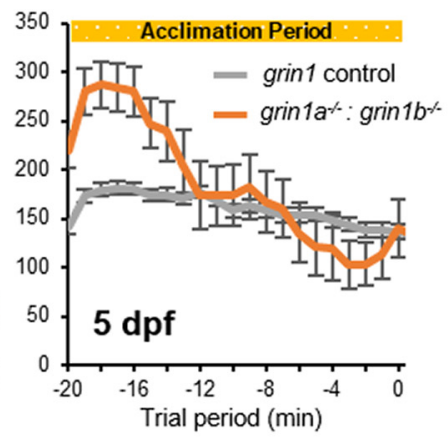

B

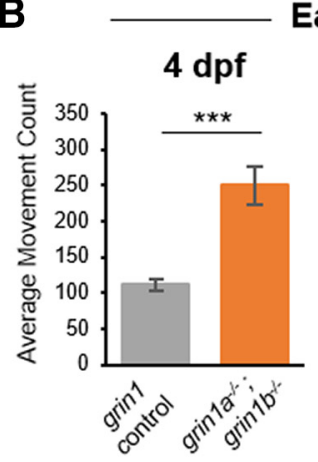

Early Acclimation
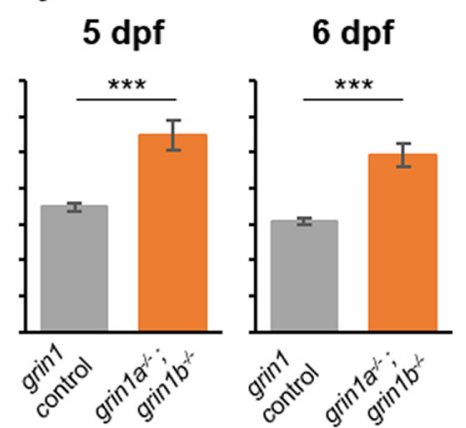

D

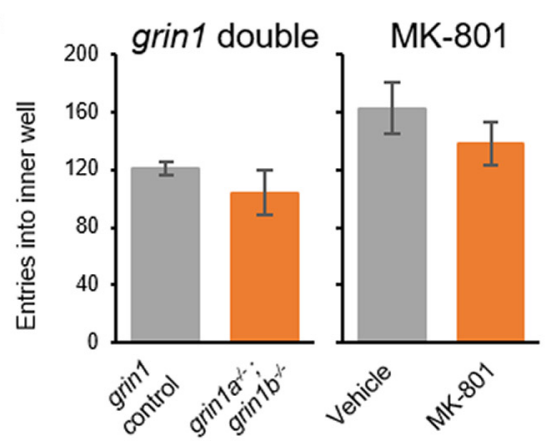

E

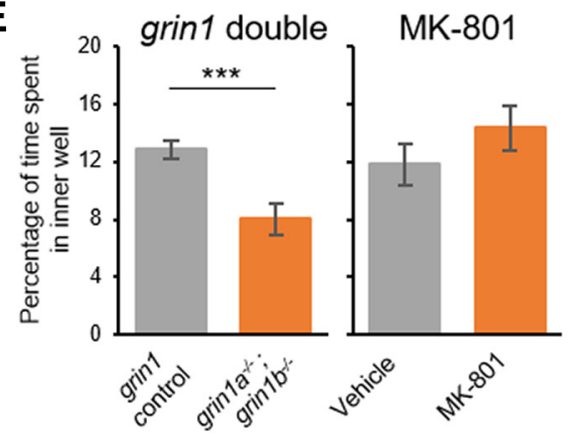

Figure 9. MK-801 cannot phenocopy all altered behaviors in grin1 double mutants. $\boldsymbol{A}$, Spontaneous movements during the acclimation period at 4 and 5 dpf. Line graphs of average number of movement counts per minute (mean \pm SEM) for grin 1 control (grin $1 a^{+/+} ;$grin $1 b^{+/+},{\text {grin } 1 a^{+/+}}_{\text {grin } 1 b^{+/-}}$and grin $1 a^{+/-} ;$grin $\left.1 b^{+/+}, n=100, n=101\right)$ or grin $1 a^{-/-} ;$grin $1 b^{-/-}$ ( $n=17, n=17)$ for 4 and $5 \mathrm{dpf}$, respectively. $\boldsymbol{B}$, Bar graphs of associated spontaneous movement trials, depicting average movements (mean \pm SEM) during the early acclimation (first 5 min of acclimation) for $4 \mathrm{dpf}\left({ }^{* * *} p=4.4 \mathrm{e}-9, t\right.$ test), $5 \mathrm{dpf}\left({ }^{* *} p=5.2 \mathrm{e}-8, t\right.$ test), and $6 \mathrm{dpf}\left({ }^{* * *} p=1.9 \mathrm{e}-12, t\right.$ test). Bar graph for $6 \mathrm{dpf}$ is for reference and is the same data as in Figure 7G. C, Schematic representation of the inner and outer portions of a behavior well. $\boldsymbol{D}, \boldsymbol{E}$, Number of entries into the inner section of behavior well (mean \pm SEM) (D) and percentage of time spent in the inner behavior well (mean \pm SEM) $(\boldsymbol{E})$ during the spontaneous movement paradigm (Fig. 7) (*** $p=4.1$ e-4 for grin1 double, $t$ test). Different conditions/genotypes tested include the following: grin1 control (grin $1 a^{+/+} ;$grin $1 b^{+/+}$, grin $1 a^{+/+} ;$grin $1 b^{+/-}$and grin $1 a^{+/-} ;$grin $1 b^{+/+}(n=148)$ or grin $1 a^{-/-} ;$grin $1 b^{-/-}(n=32)$; and vehicle (0.1\% DMSO) ( $n=26)$ or MK-801 (20 $\mu \mathrm{M}$ in 0.1\% DMSO) $(n=27)$ treated.

To further assess the light-to-dark behavioral response, we measured the distance traveled in the second after the light change. Because of the reduced responsiveness to a dark flash stimulus (Fig. 6F), we suspected the grin 1 double mutants would show deficits in responding to the extinguishing of the light. Indeed, grin 1 double mutant showed significant decreases in this photic-evoked response compared with controls, a phenotype recapitulated by MK-801 treatment (Fig. 8A). Paralleling the Obend results, grin $1 a$ and grin $1 b$ single mutants showed no altered response (data not shown).

In terms of additional swim parameters, we found that both the swim length (Fig. 8B) and swim speed (Fig. 8C) were significantly increased in both the grin 1 double-mutant and the MK801-treated fish compared with controls. grin $1 a$ and grin $1 b$ single mutants showed no alteration in these swim kinetics (data not shown). Thus, MK-801 phenocopies both gross and fine movement parameters of the grin1 double mutants, suggesting, at minimum, that these deficits reflect a lack of NMDAR-mediated transmission. In addition to these spontaneous swim parameters, MK-801 treatment of WT fish also phenocopied the prey-capture deficit found in grin1 double-mutant fish (Fig. 5B): MK801-treated fish $(0.28 \pm 0.12, n=15$; mean \pm SEM, $n=$ number of fish tested) captured a significantly lower percentage of paramecia compared and standardized to their vehicle control $(1.00 \pm 0.12, n=14)(p=9.4 \mathrm{e}-6, t$ test $)$.

Behavioral deficits in the grin1 double mutants with a likely developmental origin

The grin1 double-mutant (Fig. 7C,G), but not MK-801-treated (Fig. $7 D, H$ ), fish exhibited hyperactivity immediately after being placed in the testing apparatus. Because acute NMDAR antagonism does not replicate this hyperactivity, it is presumably developmental in origin. Consistent with this idea, grin1 doublemutant fish at earlier developmental time points (4 and $5 \mathrm{dpf}$ ) also show hyperactivity during the early acclimation period (Fig. $9 A, B)$. The lack of hyperactivity in the MK-801-treated fish is unlikely due to off-target effects of MK-801, since applying MK801 to the grin 1 double-mutant fish did not abolish this hyperactivity (data not shown). Hence, the circuit underlying the hyperactivity during the early acclimation period is most likely due to the lack of NMDAR-mediated signaling during early development.

Zebrafish larvae spend a greater duration of time in the outer portions of a circular well than would be expected by random chance (Fig. 9C; and data not shown) (Schnorr et al., 2012). Changes in place preference in larval zebrafish may reflect levels of anxiety (Schnorr et al., 2012) and/or altered search strategy (Horstick et al., 2017). While grin 1 double mutants enter the center portion of the well as often as their control siblings (Fig. 9D, left), they exit more rapidly and do not remain in the center as long as controls (Fig. 9E, left). In contrast, fish treated with MK801 showed no difference compared with control in either their entrances into the center of the well or the time spent there (Fig. $9 D, E$, right panels). Hence, variations in place preference in the grin1 double-mutant fish presumably have a developmental origin.

\section{grin1 mutants fail to habituate to acoustic stimuli}

The circuit underlying escape responses to acoustic stimuli in fish is well studied and NMDAR-dependent (Lopez-Schier, 
2019). We therefore assayed acoustic responsiveness, habituation, and sensorimotor gating to further define the breadth of behaviors grin 1 mutant fish can generate.

Intense auditory stimuli induce rapid escape responses in larvae, including Mauthner-cell-initiated Short latency Cbend (SLC) responses (Burgess and Granato, 2007b). SLC responsiveness was increased in the grin 1 double mutants compared with controls (Fig. 10A). Additional kinematics of this startle response in grin 1 double mutants, including the initial bend angle, angular velocity, and duration and latency of response, were unchanged (data not shown), suggesting that the grin1 double-mutant startle kinematics were unaltered. Paralleling the result in the grin 1 double mutant, SLC responsiveness was increased in grin $1 a^{-/-}$larvae compared with sibling controls at low- and high-stimulus intensities (Fig. 10B), whereas grin $1 b^{-1-}$ larvae showed no differences (Fig. 10C).

To test for changes in SLC habituation, we presented startling stimuli at $1 \mathrm{~s}$ intervals and measured habituation as the percent decrease in SLC responsiveness (see Materials and Methods). grin1 double mutants fish showed a decrease in habituation compared with controls (Fig. $10 D)$. Even at a lower stimulus intensity, grin1 double-mutant fish still showed a habituation deficit (data not shown), confirming that habituation was not being obscured by the high SLC response percentage. grin $1 a^{-1-}$ (Fig. 10E), but not grin $1 b^{-/-}$(Fig. 10F), larvae also exhibited decreases in habituation compared with WT siblings.

In summary, these results are consistent with previous work, which has shown that MK-801 treatment leads to both increased response to acoustic startle and decreased habituation (Wolman et al., 2011). They also suggest that acoustic startle responses and nonassociative learning are primarily mediated by GluN1a.

\section{grin1 mutants show sensorimotor gating deficits}

Despite its simplicity, the SLC response circuit exhibits a form of sensorimotor gating, PPI, which results in the attenuation of the SLC response (Burgess and Granato, 2007b). The extent of PPI depends on the ISI between the prepulse and pulse stimuli, and pharmacological blockade of NMDAR signaling selectively affects long-interval (>300 ms) PPI (Burgess and Granato, 2007b; Bergeron et al., 2015). We therefore tested PPI at 50 and $500 \mathrm{~ms}$ intervals and found that grin 1 double mutants had deficits at $500 \mathrm{~ms}$ ISI, but not at $50 \mathrm{~ms}$ ISI (Fig. $10 G)$, consistent with previous pharmacological experiments.
B
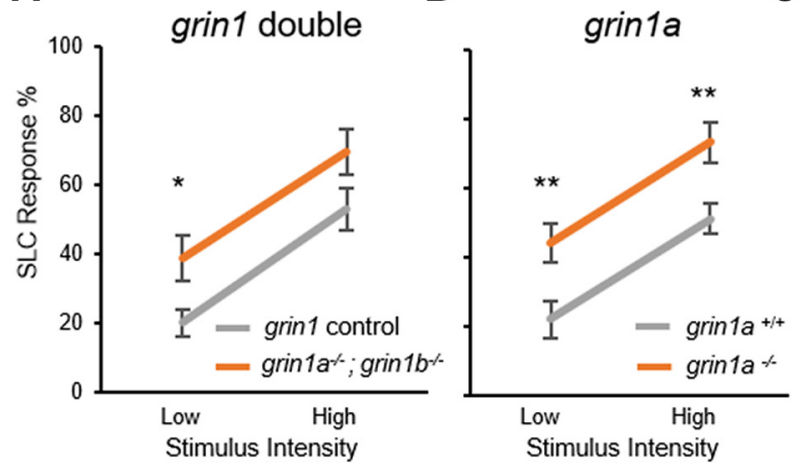

C

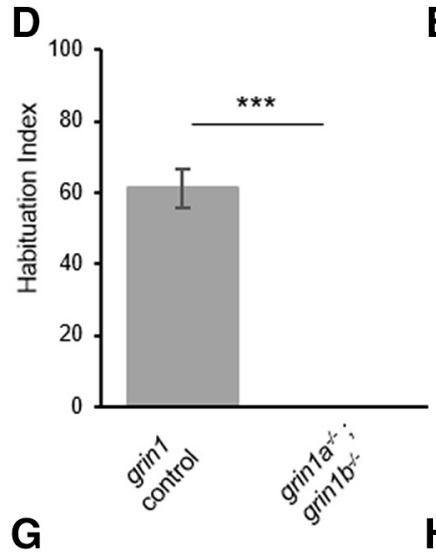

E
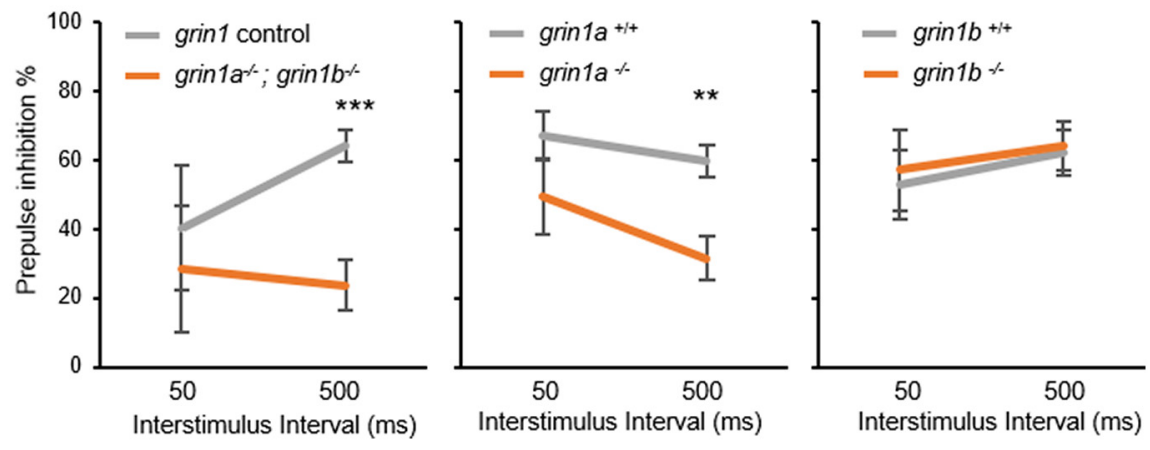

Figure 10. Disruption of sensorimotor gating and nonassociative learning in grin1 mutant fish. $\boldsymbol{A}-\boldsymbol{C}$, Responsiveness of larvae to a low- and high-intensity acoustic stimuli measured as percentage of larvae responding with a SLC for the following: (A) grin1 control (grin $1 a^{+/+} ;$grin $1 b^{-/-}$and grin $1 a^{+/-} ;$grin $\left.1 b^{-1-}, n=22\right)$ or grin1 double mutant $(n=20)$ $\left({ }^{*} p=0.019\right.$ for low intensity, $t$ test); $(\boldsymbol{B})$ grin1 $a^{+/+}(n=18)$ or grin $1 a^{-1-}(n=20) \quad\left(^{* *} p=0.0091\right.$ for low intensity; ${ }^{* *} p=0.0044$ for high intensity, $t$ test); and $(\boldsymbol{C})$ grin $1 b^{+/+}(n=13)$ or grin1 $b^{-1-}(n=15)$. D-F, Habituation to a high-intensity acoustic stimuli measured as a habituation index for the following: (D) grin1 control (grin1a ${ }^{+/+} ; g r i n 1 b^{-/-}$and

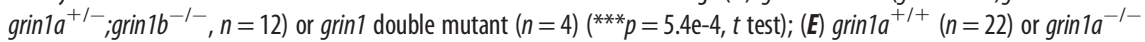
$(n=21)\left({ }^{* *} p=0.0034, t\right.$ test $)$; and $(\boldsymbol{F})$ grin $1 b^{+/+}(n=12)$ or grin $1 b^{-l-}(n=10)$. G-I, PPI percentage for ISIs of 50 and 500 ms for the following: (G) grin1 control $(n=6, n=17)$ or grin1 double $(n=8, n=12)\left({ }^{* * *} p=4.0\right.$ e-5 for long ISI, $t$

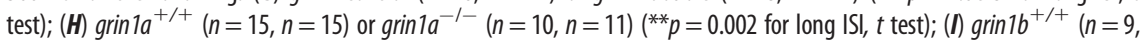
$n=9)$ or grin $1 b^{-1-}(n=10, n=12)$. We used (grin1 $a^{+1-} ;$ grin $\left.1 b^{-1-}\right)$ intercrosses to generate an enhanced number of grin 1 double mutants for $\boldsymbol{A}, \boldsymbol{D}$, and $\boldsymbol{G}$ in this assay. In these crosses, grin $1 a^{+/+} ;$grin $1 b^{-/-}$and grin $1 a^{+/-} ;$grin $1 b^{-1-}$ were used as a control, as no deficits were observed in grin $1 b^{-1-}$ fish.

Paralleling these results, grin $1 a^{-1-}$ larvae also showed deficits at $500 \mathrm{~ms}$ ISI, but not at $50 \mathrm{~ms}$ ISI (Fig. 10H), whereas grin $1 b^{-1-}$ larvae showed no differences in PPI (Fig. 10I), indicating a requirement for GluN1a in PPI at long ISI.

\section{Discussion}

NMDAR-mediated signaling underlies a variety of higher-order brain functions, including neural plasticity, learning, memory, 
and neurodevelopment and correspondingly modulates a variety of behaviors (Paoletti et al., 2013). In the present study, we took advantage of zebrafish to generate knockouts of the obligatory GluN1 subunit. Surprisingly, we find fish completely lacking NMDAR-mediated signaling can survive through early development, until $\sim 10 \mathrm{dpf}$. These larvae lacking NMDAR can initiate a wide variety of behaviors, including prey-capture, burst swimming, and stereotypic responses to visual and acoustic stimuli, although generally fish lacking NMDARs showed deficits relative to WT. In addition, using acute treatment with MK-801 as a reference, we find that some of these behavioral deficits arise from a lack of NMDAR-mediated transmission, whereas others appear to have a developmental component. Given that these NMDARlacking fish can perform an array of behaviors, they will be an invaluable tool to study how NMDAR signaling affects early brain development.

A variety of lines of evidence support the idea that grin 1 double-mutant fish have no residual GluN1 function. First, we introduced CRISPR-Cas9 lesions in the highly conserved M3 pore lining helix (Fig. $3 A-D$ ), such that any alternative splicing event that circumvented the mutations would render the channel nonfunctional. Second, we detected no WT grin 1 mRNA in the individual mutants (Fig. $3 E, F$ ), nor could we detect zGluN1a or zGluN1b protein in lysate from larval grin1 double mutants (Fig. $5 C)$. Finally, many behavioral deficits of the grin 1 double mutant were phenocopied by MK-801, including prey-capture (data not shown), the response to a photic stimulus (Figs. 7, 8), and acoustic startle modulation (Fig. 10).

Zebrafish underwent an ancient genome duplication (Amores et al., 1998), leading to two grin1 paralogs, grin1a and grin1b. These zebrafish GluN1 paralogs, when coexpressed with rat GluN2A in a heterologous expression system, show glutamategated currents and are highly $\mathrm{Ca}^{2+}$-permeable with zebrafish GluN1a and GluN1b similar to each other and to the rat GluN1 subunit (Fig. 1). Nevertheless, grin1a and grin1b showed notable differences in expression (Fig. 2). grin1a is expressed more robustly in early development than grin $1 b$, and it is expressed in the developing spinal cord, where grin $1 b$ is absent, suggesting differential spatiotemporal requirements for the two paralogs.

Despite sequence and functional similarity, zebrafish lacking either grin1a or grin1b show stark phenotypic and behavioral differences. grin $1 a^{-1-}$ fish have reduced viability and growth deficits, whereas grin $1 b^{-1-}$ adults do not (Fig. 4). Similarly, grin $1 a^{-/-}$fish have a prey-capture deficit (Fig. 6C) and an altered responsiveness to acoustic stimuli (Fig. 10), whereas grin $1 b^{-/-}$do not. Still, while we did not identify any phenotype for grin $1 b^{-1-}$ mutant alone, there were instances where the grin 1 double-mutant fish showed distinct phenotypes to the single mutants, including responses to a dark flash (Fig. $6 E-H$ ) and spontaneous and evoked behavior (Figs. 7, 8), highlighting that grin $1 b$ plays an important role in brain function. Overall, these results suggest that grin $1 a$ and grin $1 b$ have unique functions, likely due to their spatiotemporal expressional differences, but future experiments will be needed to fully explain these differences and to better understand their distinct roles in the circuits generating these behaviors.

Genetic compensation occurs in response to non-sense-mediated decay (El-Brolosy et al., 2019), but we did not detect any compensatory increases in the other paralog (Fig. 3G,H). While NMDARs have distinct functional properties, other classes of iGluRs, including AMPARs and kainate receptors, could provide some compensatory and/or redundancy of function either at the synaptic or circuit level. Consistent with this idea, activation of either AMPAR or NMDAR is sufficient to generate organized locomotive activity in spinalized zebrafish, but only AMPARs are necessary for generation of this movement (Wahlstrom-Helgren et al., 2019). This observation may account for the finding that grin $1 a$ single mutants and grin 1 double mutants generate coordinated spontaneous locomotor activity despite an apparent lack of NMDAR in their spinal cord. Nevertheless, future studies will be needed to explore compensatory mechanisms in the grin $1 \mathrm{mu}-$ tant fish.

The grin 1 double mutants were able to generate many behaviors, including sufficiently coordinated sensory and locomotor activity for prey-capture (Fig. 6B), O-bend responses to visual stimuli (Fig. 6F-H), burst swimming (Fig. 7C), and SLC responses to acoustic stimuli (Fig. 10A). Relative to WT, however, the grin 1 double mutants display a variety of alterations in their behavioral repertoire. Many of these alterations are replicated by administration of MK-801, including prey-capture deficits (data not shown), gross motor movement responses to photic stimuli (Fig. 7D), abnormal startle responsiveness (Fig. $8 A$ ), swim kinetics (Fig. $8 B, C$ ), and SLC habituation (Wolman et al., 2011). We did not determine the status of the swim bladder in all experiments involving grin1 double-mutant fish (e.g., prey-capture) and hence cannot entirely rule out the possibility that the underinflation of the swim bladder in grin1 double mutants is contributing to some of these phenotypes. Nevertheless, WT larvae treated with MK-801 retain a fully inflated bladder yet replicate many of the grin 1 double-mutant behavioral alterations. This suggests that the observed alterations are a result of the lack of NMDAR-mediated transmission, rather than caused by underinflation of the swim bladder. It is also possible that short-term block of NMDAR-mediated transmission by MK-801 may be overshadowing additional developmental roles of NMDAR, as is likely the case for more complex behaviors.

We found evidence that certain behavior alterations stem from developmental effects of loss of NMDAR-mediated transmission. Some examples are the hyperactivity at the onset of experimentation (Fig. 9A,B) and a reduced time spent in the center of the well (Fig. $9 E$ ). It is interesting to note that the alteration in visual motor response in the grin1 double mutant, notably a decrease in movements generated, has also been previously reported in WT larvae in larger behavior chambers (Horstick et al., 2017). This similarity may suggest either an alteration in photic-stimuli-induced search strategy or an alteration in the perception of their environment for the grin 1 double mutant.

In response to acoustic stimuli, the grin 1 double mutant displayed multiple phenotypes previously observed in pharmacological inhibition of NMDAR in larvae: an increase in SLC responsiveness (Fig. 10A) (Bergeron et al., 2015), a decrease in habituation to an acoustic stimuli (Fig. 10D) (Roberts et al., 2011; Wolman et al., 2011), and a deficit in PPI at long ISIs (Fig. $10 G$ ) (Burgess and Granato, 2007b; Bergeron et al., 2015). Interestingly, these phenotypes are also all observed in the grin1a single mutant, suggesting a greater role of zGluN1a in the generation of these behaviors (Fig. 10B,E,H). The acoustic startle circuit generation of SLC responses is well characterized (Lopez-Schier, 2019), and these mutants may be an ideal resource to elucidate cell specific requirements for NMDAR transmission within the circuit.

We developed a model that provides a unique opportunity to explore NMDAR functions in an early vertebrate nervous system. Given the similarity in sequence and measured 
physiological properties of the grin1 paralogs to their mammalian orthologs, and the wide array of behaviors larval zebrafish lacking all NMDAR-mediated transmission are capable of (Wahlstrom-Helgren et al., 2019), zebrafish are an ideal organism to study the developmental roles of NMDAR. We found that removing GluN1, and by extension removing NMDARmediated transmission, altered multiple behaviors. Remarkably, many complex behaviors, such as coordinated swimming and responses to stimuli, were retained in mutants lacking GluN1. This set of behaviors, as well as the viability through early larval stages, allows for future studies in defining the role of NMDAR in cell fate decisions and circuit formation in the early vertebrate nervous system.

\section{References}

Alsaloum M, Kazi R, Gan Q, Amin J, Wollmuth LP (2016) A molecular determinant of subtype-specific desensitization in ionotropic glutamate receptors. J Neurosci 36:2617-2622.

Amin JB, Leng X, Gochman A, Zhou HX, Wollmuth LP (2018) A conserved glycine harboring disease-associated mutations permits NMDA receptor slow deactivation and high $\mathrm{Ca}^{2+}$ permeability. Nat Commun 9:3748.

Amin JB, Salussolia CL, Chan K, Regan MC, Dai J, Zhou HX, Furukawa H, Bowen ME, Wollmuth LP (2017) Divergent roles of a peripheral transmembrane segment in AMPA and NMDA receptors. J Gen Physiol 149:661-680.

Amores A, Force A, Yan YL, Joly L, Amemiya C, Fritz A, Ho RK, Langeland J, Prince V, Wang YL, Westerfield M, Ekker M, Postlethwait JH (1998) Zebrafish hox clusters and vertebrate genome evolution. Science 282:1711-1714.

Bergeron SA, Carrier N, Li GH, Ahn S, Burgess HA (2015) Gsx1 expression defines neurons required for prepulse inhibition. Mol Psychiatry 20:974985.

Burgess HA, Granato M (2007a) Modulation of locomotor activity in larval zebrafish during light adaptation. J Exp Biol 210:2526-2539.

Burgess HA, Granato M (2007b) Sensorimotor gating in larval zebrafish. J Neurosci 27:4984-4994.

Burnashev N, Schoepfer R, Monyer H, Ruppersberg JP, Günther W, Seeburg PH, Sakmann B (1992) Control by asparagine residues of calcium permeability and magnesium blockade in the NMDA receptor. Science 257:1415-1419.

Chakraborty A, Murphy S, Coleman N (2017) The role of NMDA receptors in neural stem cell proliferation and differentiation. Stem Cells Dev 26:798-807.

Chang N, Sun C, Gao L, Zhu D, Xu X, Zhu X, Xiong JW, Xi JJ (2013) Genome editing with RNA-guided Cas9 nuclease in zebrafish embryos. Cell Res 23:465-472.

Cline H, Haas K (2008) The regulation of dendritic arbor development and plasticity by glutamatergic synaptic input: a review of the synaptotrophic hypothesis. J Physiol 586:1509-1517.

Cox JA, Kucenas S, Voigt MM (2005) Molecular characterization and embryonic expression of the family of N-methyl-D-aspartate receptor subunit genes in the zebrafish. Dev Dyn 234:756-766.

Dreosti E, Lopes G, Kampff AR, Wilson SW (2015) Development of social behavior in young zebrafish. Front Neural Circuits 9:39.

El-Brolosy MA, Kontarakis Z, Rossi A, Kuenne C, Gunther S, Fukuda N, Kikhi K, Boezio GL, Takacs CM, Lai SL, Fukuda R, Gerri C, Giraldez AJ, Stainier DY (2019) Genetic compensation triggered by mutant mRNA degradation. Nature 568:193-197.

Emran F, Rihel J, Dowling JE (2008) A behavioral assay to measure responsiveness of zebrafish to changes in light intensities. J Vis Exp 20:923.

Fernandes AM, Fero K, Arrenberg AB, Bergeron SA, Driever W, Burgess HA (2012) Deep brain photoreceptors control light-seeking behavior in zebrafish larvae. Curr Biol 22:2042-2047.

Forrest D, Yuzaki M, Soares HD, Ng L, Luk DC, Sheng M, Stewart CL, Morgan JI, Connor JA, Curran T (1994) Targeted disruption of NMDA receptor 1 gene abolishes NMDA response and results in neonatal death. Neuron 13:325-338.

Gahtan E, Tanger P, Baier H (2005) Visual prey-capture in larval zebrafish is controlled by identified reticulospinal neurons downstream of the tectum. J Neurosci 25:9294-9303.
Gambrill AC, Barria A (2011) NMDA receptor subunit composition controls synaptogenesis and synapse stabilization. Proc Natl Acad Sci USA 108:5855-5860.

Gan Q, Salussolia CL, Wollmuth LP (2015) Assembly of AMPA receptors: mechanisms and regulation. J Physiol 593:39-48.

Gestri G, Link BA, Neuhauss SC (2012) The visual system of zebrafish and its use to model human ocular diseases. Dev Neurobiol 72:302-327.

Hansen KB, Yi F, Perszyk RE, Furukawa H, Wollmuth LP, Gibb AJ, Traynelis SF (2018) Structure, function, and allosteric modulation of NMDA receptors. J Gen Physiol 150:1081-1105.

Horstick EJ, Bayleyen Y, Sinclair JL, Burgess HA (2017) Search strategy is regulated by somatostatin signaling and deep brain photoreceptors in zebrafish. BMC Biol 15:4.

Huettner JE, Bean BP (1988) Block of N-methyl-D-aspartate-activated current by the anticonvulsant MK-801: selective binding to open channels. Proc Natl Acad Sci USA 85:1307-1311.

Hunt DL, Castillo PE (2012) Synaptic plasticity of NMDA receptors: mechanisms and functional implications. Curr Opin Neurobiol 22:496-508.

Hwang WY, Fu Y, Reyon D, Maeder ML, Tsai SQ, Sander JD, Peterson RT, Yeh JR, Joung JK (2013) Efficient genome editing in zebrafish using a CRISPR-Cas system. Nat Biotechnol 31:227-229.

Jatzke C, Watanabe J, Wollmuth LP (2002) Voltage and concentration dependence of $\mathrm{Ca}^{2+}$ permeability in recombinant glutamate receptor subtypes. J Physiol 538:25-39.

Kazi R, Dai J, Sweeney C, Zhou HX, Wollmuth LP (2014) Mechanical coupling maintains the fidelity of NMDA receptor-mediated currents. Nat Neurosci 17:914-922.

Kutsuwada T, Sakimura K, Manabe T, Takayama C, Katakura N, Kushiya E, Natsume R, Watanabe M, Inoue $\mathrm{Y}$, Yagi T, Aizawa S, Arakawa M, Takahashi T, Nakamura Y, Mori H, Mishina M (1996) Impairment of suckling response, trigeminal neuronal pattern formation, and hippocampal LTD in NMDA receptor epsilon 2 subunit mutant mice. Neuron 16:333-344.

Lopez-Schier H (2019) Neuroplasticity in the acoustic startle reflex in larval zebrafish. Curr Opin Neurobiol 54:134-139.

Menezes FP, Kist LW, Bogo MR, Bonan CD, Da Silva RS (2015) Evaluation of age-dependent response to NMDA receptor antagonism in zebrafish. Zebrafish 12:137-143.

Paoletti P, Bellone C, Zhou Q (2013) NMDA receptor subunit diversity: impact on receptor properties, synaptic plasticity and disease. Nat Rev Neurosci 14:383-400.

Roberts AC, Reichl J, Song MY, Dearinger AD, Moridzadeh N, Lu ED, Pearce K, Esdin J, Glanzman DL (2011) Habituation of the C-start response in larval zebrafish exhibits several distinct phases and sensitivity to NMDA receptor blockade. PLoS One 6:e29132.

Salussolia CL, Corrales A, Talukder I, Kazi R, Akgul G, Bowen M, Wollmuth LP (2011) Interaction of the M4 segment with other transmembrane segments is required for surface expression of mammalian alpha-amino-3hydroxy-5-methyl-4-isoxazolepropionic acid (AMPA) receptors. J Biol Chem 286:40205-40218.

Salussolia CL, Gan Q, Kazi R, Singh P, Allopenna J, Furukawa H, Wollmuth LP (2013) A eukaryotic specific transmembrane segment is required for tetramerization in AMPA receptors. J Neurosci 33:9840-9845.

Schmidt JT, Buzzard M, Borress R, Dhillon S (2000) MK801 increases retinotectal arbor size in developing zebrafish without affecting kinetics of branch elimination and addition. J Neurobiol 42:303-314.

Schnorr SJ, Steenbergen PJ, Richardson MK, Champagne DL (2012) Measuring thigmotaxis in larval zebrafish. Behav Brain Res 228:367-374.

Seibt KJ, Piato AL, da Luz Oliveira R, Capiotti KM, Vianna MR, Bonan CD (2011) Antipsychotic drugs reverse MK-801-induced cognitive and social interaction deficits in zebrafish (Danio rerio). Behav Brain Res 224:135139.

Semmelhack JL, Donovan JC, Thiele TR, Kuehn E, Laurell E, Baier H (2014) A dedicated visual pathway for prey detection in larval zebrafish. Elife 3:e04878.

Sison M, Gerlai R (2011a) Behavioral performance altering effects of MK-801 in zebrafish (Danio rerio). Behav Brain Res 220:331-337.

Sison M, Gerlai R (2011b) Associative learning performance is impaired in zebrafish (Danio rerio) by the NMDA-R antagonist MK-801. Neurobiol Learn Mem 96:230-237.

Sprengel R, Suchanek B, Amico C, Brusa R, Burnashev N, Rozov A, Hvalby O, Jensen V, Paulsen O, Andersen P, Kim JJ, Thompson RF, Sun W, 
Webster LC, Grant SG, Eilers J, Konnerth A, Li J, McNamara JO, Seeburg PH (1998) Importance of the intracellular domain of NR2 subunits for NMDA receptor function in vivo. Cell 92:279-289.

Swain HA, Sigstad C, Scalzo FM (2004) Effects of dizocilpine (MK-801) on circling behavior, swimming activity, and place preference in zebrafish (Danio rerio). Neurotoxicol Teratol 26:725-729.

Thisse C, Thisse B, Schilling TF, Postlethwait JH (1993) Structure of the zebrafish snaill gene and its expression in wild-type, spadetail and no tail mutant embryos. Development 119:1203-1215.

Traynelis SF, Wollmuth LP, McBain CJ, Menniti FS, Vance KM, Ogden KK, Hansen KB, Yuan H, Myers SJ, Dingledine R (2010) Glutamate receptor ion channels: structure, regulation, and function. Pharmacol Rev 62:405496.

Wahlstrom-Helgren S, Montgomery JE, Vanpelt KT, Biltz SL, Peck JH, Masino MA (2019) Glutamate receptor subtypes differentially contribute to optogenetically activated swimming in spinally transected zebrafish larvae. J Neurophysiol 122:2414-2426.

Watanabe J, Beck C, Kuner T, Premkumar L, Wollmuth LP (2002) DRPEER: a motif in the extracellular vestibule conferring high $\mathrm{Ca}^{2+}$ flux rates in NMDA receptor channels. J Neurosci 22:10209-10216.

Wollmuth LP (2018) Ion permeation in ionotropic glutamate receptors: still dynamic after all these years. Curr Opin Physiol 2:36-41.

Wollmuth LP, Sobolevsky AI (2004) Structure and gating of the glutamate receptor ion channel. Trends Neurosci 27:321-328.

Wolman MA, Jain RA, Liss L, Granato M (2011) Chemical modulation of memory formation in larval zebrafish. Proc Natl Acad Sci USA 108:15468-15473.

Yelshansky MV, Sobolevsky AI, Jatzke C, Wollmuth LP (2004) Block of AMPA receptor desensitization by a point mutation outside the ligandbinding domain. J Neurosci 24:4728-4736. 